\title{
The stipitate species of Hypocrea (Hypocreales, Hypocreaceae) including Podostroma
}

\author{
HOLLY L. CHAMBERLAIN, AMY Y. ROSSMAN, ELWIN L. STEWART, TAUNO ULVINEN \\ and GARY J. SAMUELS
}

CHAMBERLAIN, H. L., ROSSMAN, A. Y., STEWART, E. L., ULVINEN, T. \& SAMUELS, G. J. 2004: The stipitate species of Hypocrea (Hypocreales, Hypocreaceae) including Podostroma. - Karstenia 44: 124. 2004. Helsinki. ISSN 0453-3402.

\begin{abstract}
Stipitate species of Hypocrea have traditionally been segregated as the genus Podostroma. The type species of Podostroma is P. leucopus for which $P$. alutaceum has been considered an earlier synonym. Study of the type and existing specimens suggests that these two taxa can be distinguished based on morphology and biology. Podostroma leucopus is herein recognized as Hypocrea leucopus (P. Karst.) H. Chamb., comb. nov., thus Podostroma is a synonym of Hypocrea. The genus Podocrea, long considered a synonym of Podostroma, is based on Sphaeria alutacea, a species that is recognized as $H$. alutacea. A neotype is designated for Sphaeria alutacea. Both $H$. alutace $a$ and $H$. leucopus are redescribed and illustrated. The new species $H$. nybergiana T. Ulvinen \& H. Chamb., spec. nov. is described and illustrated. In addition to H. leucopus, seven species of Podostroma are transferred to Hypocrea, viz. H. africana (Boedijn) H. Chamb., comb. nov., H. cordyceps (Penz. \& Sacc.) H. Chamb., comb. nov., H. daisenensis (Yoshim. Doi \& Uchiy.) H. Chamb., comb. nov., H. eperuae (Rogerson \& Samuels) H. Chamb., comb. nov., H. gigantea (Imai) H. Chamb., comb. nov., H. sumatrana (Boedijn) H. Chamb., comb. nov., and H. truncata (Imai) H. Chamb., comb. nov. A key to the 17 species of stipitate Hypocrea including Podostroma and Podocrea is presented.
\end{abstract}

Key words: Ascomycetes, Hypocreaceae, Hypocreales, Podocrea, systematics, Trichoderma

H. L. Chamberlain and E.L. Stewart, Department of Plant Pathology, 3086 Buckhout Laboratory, The Pennsylvania State University, University Park, PA 16802, USA

A.Y. Rossman and G.J. Samuels, United States Department of Agriculture, Agricultural Research Service, Systematic Botany and Mycology Laboratory, Rm. 304, B-011A, Beltsville, MD 20705-2350, USA

T. Ulvinen, Kannuskuja 1 DI, FI-90540 Oulu, Finland

\section{Introduction}

Species of Hypocrea Fr. that have a stipe, especially those with a clavate to cylindrical stroma, traditionally have been segregated as the genus Podostroma P. Karst. (Karsten 1892, Doi 1967, Imai 1932, Rogerson \& Samuels 1992, Rossman et al. 1999, Seaver \& Chardón 1926). Podostroma was described for members of the Hypocreaceae having stipitate, clavate, erect, fleshy, brightcolored stromata within which perithecial as- comata are immersed. Originally Podostroma was described with only one species, P. leucopus P. Karst. (Karsten 1892). Later Podocrea Lindau (1897), based on P. alutacea (Pers. : Fr.) Lindau, was proposed for species having many of the same characteristics as Podostroma. Atkinson (1905) and authors since then (Clements \& Shear 1931, Rossman et al. 1999) have regarded Podostroma leucopus to be synonymous with $P$. aluta- 
ceum (Pers. : Fr.) G.F. Atk., thus Podocrea has long been considered a synonym of Podostroma. No comprehensive account of Podostroma exists, but several authors have published partial taxonomic treatments of the genus (Boedijn 1934, 1938, Doi 1966, 1967, 1973, Doi \& Uchiyama 1987, Imai 1932, Seaver \& Chardón 1926).

Considerable confusion has surrounded the type species of Podostroma, P. alutaceum, and its assumed synonym, $P$. leucopus. Karsten (1892) stated that $P$. leucopus was insecticolous but no evidence exists to support this claim. No species of Podostroma is known to be entomopathogenic although there is one insecticolous species of Hypocrea, viz. H. dipterobia Samuels \& Rogerson (Samuels \& Rogerson 1986). The synonymy of $P$. leucopus with $P$. alutaceum, first proposed by Atkinson (1905), has been accepted since then. For this study the type specimens of P. leucopus and Sphaeria alutacea Pers. : Fr. were examined as were numerous specimens identified as $P$. alutaceum. Based on morphological and biological characteristics, these two taxa are recognized as distinct species.

The stroma of species included in Podostro$m a$ is typically light in color, at least when fresh, and is fleshy. The centrum is typically hypocrealean, having apical paraphyses that dissolve at maturity. The asci and ascospores of Podostro$m a$ are indistinguishable from those of Нypocrea. The asci are cylindrical and the apex is only slightly thickened and has an obscure pore. The ascospores are hyaline, spinulose and bicellular. The two halves of each ascospore disarticulate early in development, giving the appearance that there are 16 spores in each ascus (Rossman et al. 1999). Podostroma is distinguished from Hypocrea only on the basis of gross morphology of the ascomata. The stipitate stromata of $P$. alutaceum and $P$. leucopus are macroscopically different from the type of Hypocrea, H. rufa (Pers. : Fr.) Fr., the stroma of which is broadly attached, appearing to be effused, becoming pulvinate.

Trichoderma Pers. or verticillium-like anamorphs have been attributed to some species included in Podostroma. Doi (1966) described a verticillium-like anamorph with hyaline conidia for P. alutaceum. Doi (1967) linked P. cordyceps (Penz. $\&$ Sacc.) Yoshim. Doi and $P$. cornu-damae (Pat.) Boedijn to a Trichoderma anamorph with pale green conidia and P. solmsii (E. Fisch.) Imai to a verticillium-like anamorph with hyaline conidia.
Later Doi (1973) linked P. cornu-damae to a verticillium-like anamorph with subglobose, green conidia and in the same paper he attributed a verticillium-like anamorph with hyaline conidia to $P$. giganteum Imai. Samuels \& Lodge (1996) described typical Trichoderma anamorphs for the turbinate species $H$. brevipes (Mont.) Sacc.) ( $\equiv P$. brevipes (Mont.) Seaver), H. poronioidea A. Möller (= . orbiculare Chardón) and $H$. capitata Samuels.

Neither P. alutaceum nor P. leucopus have been subjected to DNA sequence analysis. However, Chaverri and Samuels (2003) found that one unidentified, and probably undescribed, species of Podostroma with clavate stroma is derived from within Hypocrea, closely related to Trichoderma minutisporum Bissett. In the same paper they found that Hypocrea pezizoides Berk. \& Broome, a discoidal species that has a minute central stipe, is phylogenetically close to $H$. rufalT. viride. These results argue that the stipitate habit is not phylogenetically informative at the genus level in the Hypocreaceae.

Based on the similarities of microscopic and anamorphic characteristics, the type species of Podostroma, P. leucopus, and the type species of Podocrea, $P$. alutacea, are considered members of the genus Hypocrea. In the present paper the synonymy of these species is investigated and a new species is described. Species of $\mathrm{PO}$ dostroma for which specimens are available are redescribed. A key is presented to seventeen stipitate Hypocrea species including those previously in Podostroma and Podocrea.

\section{Materials and methods}

\section{Collection and preservation of material}

Collection data and place of deposition for freshly collected material and herbarium specimens examined representative of their geographic distribution are listed after each species. Abbreviations of herbaria are taken from Holmgren et al. (1990).

\section{Cultures}

Single ascospore isolations were made with the use of a micromanipulator where possible. Perithecia were crushed in a drop of $0.7 \%(\mathrm{w} / \mathrm{v})$ sodium acetate; perithecial contents were picked up with a capillary tube and put onto cornmeal dextrose agar (CMD, Difco cornmeal agar $+2 \%$ dextrose). Ascospores were isolated when they had germinated. In other cases, following surface sterilization with alcohol, a portion of the inner stroma was 
aseptically removed with a scalpel and the tissue placed onto CMD. Cultures of Podostroma species were also obtained from the Centraalbureau voor Schimmelcultures (CBS) and Canadian Collection of Fungal Cultures (DAOM).

\section{Phenotype analysis}

Stromata from herbarium specimens were rehydrated in a drop of $3 \%$ aqueous $\mathrm{KOH}$. Reaction of stroma tissue to $\mathrm{KOH}$ was noted. Sections of stromata ca. $15 \mu \mathrm{m}$ thick were made with a cryostat (International Equipment Co., Needham Heights, MA). Whole stromata or parts of stromata, were supported for sectioning by Tissue-Tek O.C.T. Compound (Miles Inc., Elkhart, IN). Sections of stromata were observed and photographed. Colors are taken from Kornerup and Wanscher (1978). Continuous measurements were made using the Scion Image 1.0 software package (Scion Corp., Frederick, MD). Where possible, thirty objects (e.g. asci, part-spores, etc.) were measured for each morphological character for each collection; it was rarely possible to measure thirty stromata or perithecia for any collection. All observations and measurements of asci, ascospores and anamorph morphological characters were made either from wet mounts in $\mathrm{KOH}$ or water. Means and standard deviations (SD) of the measurements of all collections were calculated and were computed using the Microsoft Excel 97-SR1 or Systat 10 (SPSS 2000). Measurements are reported by one of two methods. When fewer than ten objects were measured, the total range of the object is given. When more than ten objects were measured, the number of measured objects $(\mathrm{N})$ is given and the measurements are recorded as extremes in brackets separated by the mean plus and minus the standard deviation.

Anamorph characters were taken from cultures grown on CMD for one wk or less at $20^{\circ} \mathrm{C}$ and $25^{\circ} \mathrm{C}$, with alternating darkness and cool white fluorescent light. For microscopic examination, material was first wetted in a drop of $\mathrm{KOH}$, which was replaced by water as the $\mathrm{KOH}$ evaporated. Thirty of each object for each culture were measured. Colony characters and growth rate were recorded from cultures grown on CMD and potato dextrose agar (PDA, Difco); pigment production was described from cultures grown on PDA. Growth rates were determined as follows. An actively growing culture was established on CMD. Before sporulation, $5 \mathrm{~mm}$ diam plugs were taken from the edge of the colony and placed, mycelium down, ca. $1.5 \mathrm{~cm}$ from the edge of freshly prepared PDA and CMD in vented plastic $10 \times 15 \mathrm{~mm}$ Petri dishes containing $20 \mathrm{~mL}$ of freshly prepared medium. These were incubated in darkness at 15, 20, 25, 30, and $35^{\circ} \mathrm{C}$. Cultures were measured every $24 \mathrm{~h}$ for $7 \mathrm{da}$ ( $168 \mathrm{~h}$ total). Colony radii were measured from the edge of the disc of inoculum to the edge of the growing colony.

Four types of lighting were used for microscopy, viz. brightfield (BF), brightfield phase contrast (PC), Nomarski differential interference microscopy (DIC), and fluorescence microscopy (FL). Preparations studied for fluorescence microscopy were prepared by flooding preparations that had been used for measurements with Calcofluor (Sigma Fluorescent Brightener 28, C.I. 40622 Calcofluor white M2 in a 2 molar phosphate buffer at $\mathrm{pH}$ 8.0).

\section{Results}

Most of the species studied possessed characters typical of Hypocrea including stromatic ascomata, cylindrical asci, and hyaline, spinulose, bicellular ascospores that disarticulated early in development into part-ascospores. Only ascospores of $H$. solmsii f. octospora did not disarticulate; they remained unicellular and appeared to have a blunt apiculus at each end. This species is treated below as a 'rejected species.' Podostoma and Podocrea could not be distinguished from each other; both are considered to be synonymous with Hypocrea. Species of these genera are recognized in or transferred to Hypocrea. The seventeen species of stipitate Hypocrea many of which had previously been included in Podostroma or Podocrea are distinguished in the key.

For Podostroma alutaceum and P. leucopus numerous Northern Hemisphere collections were studied. One group of collections form stromata on decaying wood while stromata of the second group arise from the ground. Although we were able to obtain cultures for only a few collections, there was a close correlation between substratum and anamorph. The anamorph of terricolous collections was verticillium-like with hyaline conidia, while conidia of the lignicolous specimens are green and the conidiophores are more irregularly branched and trichoderma-like. These differences coincide with the type specimens of $P$. leucopus and $P$. alutaceum respectively and serve to separate these two species.

The terricolous Northern Hemisphere specimens could also be divided into two groups based on the characters of their stromata and ascospores. In one group the stroma was $3-5 \mathrm{~cm}$ tall and beige. In contrast to the first group, stromata in the second group are much more robust, $2-15 \mathrm{~cm}$ tall and sometimes shallowly oncebranched at the apex; they have red-brown to brown-orange scales on the stipe. Ascospore measurements coincide with stroma size. In the first group the distal part-ascospores are subglobose to conic, 2.5-3.0 $\mu \mathrm{m}$ diam, and the proximal part-ascospores are cuneate to ellipsoidal, 3.0 $4.0 \times 2.0-3.0 \mu \mathrm{m}$. Distal part-ascospores in the second group are globose to subglobose, 3.5$4.5 \times 3.2-4.0 \mu \mathrm{m}$, and the proximal part-ascospores are ellipsoidal to wedge-shaped, 3.7-5.0 $\times 3.0-3.5$ $\mu \mathrm{m}$. Specimens having the former phenotype were collected in northern Europe and eastern North 
America. Specimens of the second group were found in northern Europe (Finland, Sweden). Stromata of the first type conform to the type specimen of $\mathrm{H}$. leucopus. For the second group, we describe a new species.

\section{Discussion}

The most important characters for distinguishing species of stipitate Hypocrea include substratum, gross morphology, color of the stroma, internal anatomy of the stroma, extent of the fertile region of the stroma, pigmentation in the surface of the stroma, and ascospore shape, size and ornamentation. According to Samuels and Lodge (1996) there is nearly continuous variation in stromatal form within Hypocrea. Within Hypocrea stromata may vary from indefinitely effused, pulvinate but broadly attached at a central point, to stipitate, clavate. In four species of Hypocrea, H. brevipes (Mont.) Sacc., H. capitata Samuels, $H$. pezizoides Berk. \& Broome, and H. poronioidea A. Möller, the fertile part of the stromata forms an expanded cap that is sharply delimited from the sterile stipe (Liu \& Doi 1995; Samuels \& Lodge 1996). Among stipitate species of Hypocrea, the base of the stroma is sterile to some degree. In $H$. leucopus the stroma is typically strongly constricted where the fertile part meets the sterile base and the sterile part comprises less than $50 \%$ of the total length of the stroma. In most species of stipitate Hypocrea, the sterile part comprises more than $50 \%$ of the total length of the stroma and the sterile base is not sharply delimited from the fertile upper part of the stroma. Several species of stipitate Hypocrea including $H$. africana (Boedijn) H. Chamb., H. alutacea, H. eperuae (Rogerson \& Samuels) H. Chamb., and H. grossa Berk. have clavate stromata where the fertile part is not at all or only slightly differentiated from the sterile base. In general the stipitate species of Hypocrea are microanatomically the same as other species of Hypocrea with the exception of $H$. brevipes and $H$. eperuae in which the stroma in longitudinal section consists of elongated, brick-like cells (Rogerson \& Samuels 1992, Samuels \& Lodge 1996). Rogerson and Samuels (1992) suggest that these brick-like cells resulted from the extension of the stroma and do not indicate any relationship between these two species.

Anamorphic states are useful in determining relationships among genera and species of the Hypocreales (Samuels \& Seifert 1987, Rossman 2000). Few species of stipitate Hypocrea species have been grown in pure culture and linked to anamorphs. Doi (1967, 1973), Liu and Doi (1995) and Tubaki (1958) have connected stipitate Hypocrea species to typical Trichoderma as well as to verticillium-like Trichoderma anamorphs. As part of this research Trichoderma and verticillium-like Trichoderma anamorphs were obtained for $H$. alutace $a$ and H. leucopus (Figs. 28-37), confirming their relationship to Hypocrea. Samuels and Lodge (1996) linked two stipitate Hypocrea, $H$. brevipes and $H$. poronioidea, to Trichoderma anamorphs that they tentatively assigned to Trichoderma sect. Pachybasium (Sacc.) Bissett (Bissett 1991). The anamorph of H. pezizoides has very pale green conidia and verticillium-like conidiophores (Liu \& Doi 1995). 


\section{Taxonomy}

Seventeen species of stipitate Hypocrea including those previously placed in Podostroma and Podocrea are included in the following key. Descriptions are provided for species with available type or authentic material as indicated in boldface. A list of excluded or doubtful species is found at the end.

\section{Key to stipitate Hypocrea including Podostroma and Podocrea}

1. Stromata with an expanded cap greater than twice diameter of stipe and sharply delimited from stipe; tropical species

1. Stromata clavate or cylindrical, fertile part, at most, only slightly broader than stipe; temperateand tropical

2. Stromata discoidal, 6-16 $\mathrm{mm}$ diam, purplish red to reddish orange, stipe inconspicuous or lacking; distal part-ascospores obovate-subglobose, 4.3-4.5 × 3.5-4.2 $\mu \mathrm{m}$, proximal part-spores obovateellipsoid, 4.8-6.7 × 3.5-4.2 $\mu \mathrm{m}$; Asia (Liu \& Doi 1995) ...................... H. pezizoides Berk. \& Broome 2. Stromata convex, in shades of brown, stipe conspicuous; New or Old World ...................... 3

3. Cap slightly tuberculate, $1.5-3 \mathrm{~mm}$ diam; distal part-ascospores globose to subglobose, $2.0-2.7 \times$ 1.9-2.6 $\mu \mathrm{m}$, proximal part-ascospores oblong to narrowly wedge-shaped, $2.4-3.0 \times 1.8-2.2 \mu \mathrm{m}$; South America (Samuels \& Lodge 1996) .................................................. H. capitata Samuels

3. Cap plane, 2-8 mm diam; distal part-ascospores $2.6-4.3 \times 1.9-3.9 \mu \mathrm{m}$, proximal part-ascospores $2.5-5.0 \times 1.7-3.2 \mu \mathrm{m}$

4. Cap 2-8 mm diam, dark to greyish brown; distal part-ascospores subglobose to conic, $3.5-4.3 \times$ 3.1-3.9 $\mu \mathrm{m}$, proximal part-spores wedge-shaped, 3.6-5.0 $\times 2.2-3.2 \mu \mathrm{m}$; on wood; South America (French Guiana), Puerto Rico, New Guinea (Samuels \& Lodge 1996), Europe, and Japan (Doi 1975) H. brevipes (Mont.) Sacc.

4. Cap 3.0-6.5 mm diam, brown, yellowish-brown, pale brown or brownish-gray; distal partascospores globose to subglobose, 2.6-2.9 × 1.9-2.5 $\mu \mathrm{m}$, proximal part-ascospores oblong, 2.5-3.5 × 1.7-2.0 $\mu \mathrm{m}$; on wood and bark; pantropical (Samuels \& Lodge 1996)

H. poronioidea A. Möller (= Podostroma orbiculare Chardón)

5. Stromata arising from 'eggs' of Phallales 6

5. Arising from leaves, seed pods, wood, or ground 7

6. Ascospores bicellular, disarticulating into two part-ascospores; distal part-ascospores ovoidal, 5.0-5.5 × 3.2-3.7 $\mu \mathrm{m}$, proximal part-ascospores 3.7-5.0 × 3.2-3.7 $\mu \mathrm{m}$; Indonesia, Japan (Boedijn 1934, Doi 1967) H. solmsii (E. Fish.) Imai

6. Ascospores unicellular, remaining whole, 10-16 $\times 4.5-5.5 \mu \mathrm{m}$

Podostroma solmsii f. octosporum, see excluded species below.

7 On decaying leaves or seed pods, possibly endophytic on specific hosts

7. On wood or ground 8

8. Arising from pods of the legume tree Eperua; stromata narrowly clavate to filiform, $15-35 \mathrm{~mm}$ tall, fertile portion 5-12 mm long, cylindrical to slightly flattened, pale brown to brown or brownish gray; distal part-ascospores subglobose to broadly ovoidal, $4-5.3 \times 3.2-4.4 \mu \mathrm{m}$, proximal partascospores wedge-shaped, 5-6.2 × 2.7-3.8 $\mu \mathrm{m}$; French Guiana, Guyana

Hypocrea eperuae (Rogerson \& Samuels) H. Chamb., comb. nov.

Basionym: Podostroma eperuae Rogerson \& Samuels, Brittonia 44: 259. 1992.

8. On fallen leaves of Fagus crenata; stromata $5-6 \mathrm{~cm}$ tall, $0.3 \mathrm{~cm}$ diam; brownish-orange with tinge of red, lower stipe pale pinkish; distal part-ascospores subglobose to ovoidal, 3.1-3.7 $\times$ 2.8-3.4 $\mu \mathrm{m}$, proximal part-ascospores ovoidal to subcylindric, 3.2-4.6 $\times 2.2-2.9 \mu \mathrm{m}$; China, Japan, Java ................................... Hypocrea cordyceps (Penz. \& Sacc.) H. Chamb., comb. nov. Basionym: Podocrea cordyceps Penz. \& Sacc., Malpighia 15: 229. 1901 (Penzig \& Saccardo 1904: 52, Pl. 36: 4, as Podocrea cordyceps; Doi 1967 as Podostroma cordyceps) 
9. Stromata long-cylindric, brownish-olive to black, 3-15 cm tall, $1.5-7.5 \mathrm{~mm}$ diam; distal partascospores 6.2-7.6 $\times 4-5 \mu \mathrm{m}$, proximal part-ascospores 7.0-9.2 $\times 3.7-4.5 \mu \mathrm{m}$; on soil, possibly

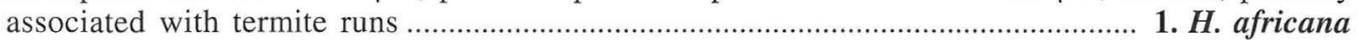

9. Stromata pale luteous, yellow, orange, red, to brown or black; part-ascospores less than $6 \mu \mathrm{m}$ diam 10

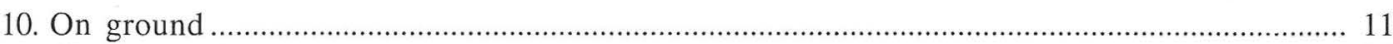

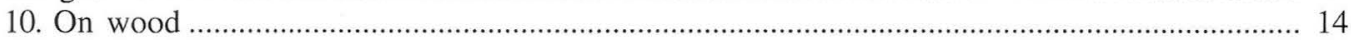

11. Stromata mostly simple, clavate or branched at apex; north temperate .................................... 12 11. Stromata cylindrical, palmately lobed or dichotomously branched from base; SE Asia and

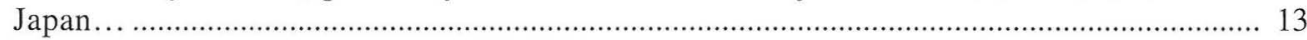

12. Stromata mostly simple, clavate, fertile part often conspicuously swollen, generally with a clear distinction between fertile and sterile parts, $1.5-8.0 \mathrm{~cm}$ tall, $0.5-5.0 \mathrm{~cm}$ diam; fertile part pale yellow to golden brown in mature specimens; stipe white to beige; distal part-ascospores $2.5-3.0 \mu \mathrm{m}$ diam, proximal part-ascospores 3.0-4.0 × 2.0-3.0 $\mu \mathrm{m}$; north temperate .....

6. H. leucopus

12. Stromata simple, clavate, reddish-brown to brownish orange, stipe white to beige, with conspicuous scales of rust pigment between area of fertile and sterile stipe, $2.2-22 \mathrm{~cm}$ tall, $1.0-6.5$ $\mathrm{mm}$ diam; distal part-ascospores 3.5-4.5 × 3.2-4.0 $\mu \mathrm{m}$, proximal part-ascospores 3.7-5.0 × 3.0$3.5 \mu \mathrm{m}$; northern Europe.

7. H. nybergiana

13. Stromata buff-orange, cylindrical, narrowly clavate to flabelliform or antler-shaped, dichotomously branched, 7-9 cm tall; distal part-ascospores 3.5-4.0 $\times 3.0-3.2 \mu \mathrm{m}$, proximal part-ascospores 3.2$4.0 \times 2.5-3.5 \mu \mathrm{m}$; trichoderma-like anamorph, olivaceous green conidia; SE Asia.

3. H. cornu-damae

13. Stromata cream-carnose, dichotomously branched from base, twisted, $5-18 \mathrm{~cm}$ tall, $3-7 \mathrm{~mm}$ diam, ostioles not distinct by naked eye; distal part-ascospores $2.7-3.3 \times 2.3-3.1 \mu \mathrm{m}$, proximal part-ascospores 3.0-4.1 × 2.2-2.9 $\mu \mathrm{m}$

4. H. daisenensis

14. Part-ascospores monomorphic, subglobose, 4-5 $\mu \mathrm{m}$ diam; stromata clavate or cylindrical with truncate, depressed apex, 1.5-3.0 $\mathrm{cm}$ tall, $5 \mathrm{~mm}$ diam; on wood; Japan

Hypocrea truncata (Imai) H. Chamb., comb. nov.

Basionym: Podostroma truncatum Imai, Trans. Sapporo Nat. Hist. Soc. 12: 117. 1932.

14. Part-ascospores dimorphic 15

15. Part-ascospores $5 \mathrm{~mm}$ or more; anamorph verticillium-like .......................................................... 16

15. Part-ascospores less than $5 \mu \mathrm{m}$; anamorph Trichoderma or unknown ............................... 17

16. Stromata yellowish brown, reddish brown to violet brown, often nearly black when dry, flatened, simple or branched, mostly without distinct stipe, $3.0-17.0 \mathrm{~cm}$ tall, $0.5-1.5 \mathrm{~cm}$ diam; distal partascospores 4.0-6.7 $\times 4.0-5.5 \mu \mathrm{m}$, proximal part-ascospores $4.2-6.5 \times 3.7-4.7 \mu \mathrm{m}$, warted; on wood

5. H. grossa

16. Stromata often bi-lobed, $\tan , 10-16 \mathrm{~cm}$ tall, $10-30 \mathrm{~mm}$ diam; ascospores globose to subglobose, 5-6 × $5 \mu \mathrm{m}$; verticillium-like anamorph, hyaline conidia; on wood; Japan

H. gigantea (Imai) H. Chamb., comb. nov.

Basionym: Podostroma giganteum Imai, Trans. Sapporo Nat. Hist. Soc.

12: 116.1932.

17. Stromata single, cylindric, pale yellowish brown, $9-14 \mathrm{~cm}$ tall, $1-2 \mathrm{~cm}$ diam, dark ostioles distinctly protruding; distal part-ascospores cuboidal, 2.5-4.0 $\mu \mathrm{m}$ diam, proximal part-ascospores elongate, 4.0-5.0 × 2.5-3.0 $\mu \mathrm{m}$; Indonesia. Hypocrea sumatrana (Boedijn) H. Chamb., comb. nov.

Basionym: Podostroma sumatranum Boedijn, Bull. Jard. Bot. Buitenzorg, ser. 3, 13: 271. 1934.

17. Stromata single or gregarious; clavate to grossly lobed or branched, sometimes fused, $0.5-8.0$ $\mathrm{cm}$ tall, $0.5-1.8 \mathrm{~cm}$ diam, golden yellow, coffee to linoleum brown, if stipe visible, white to beige, ostioles visible as darker brown against a pale brown background; distal part-ascospores $2.7-$ $3.7 \times 2.5-3.5 \mu \mathrm{m}$, proximal part-ascospores 3.0-4.0 × 2.2-2.7 $\mu \mathrm{m}$; Trichoderma anamorph, pale green conidia; on wood

2. H. alutacea 


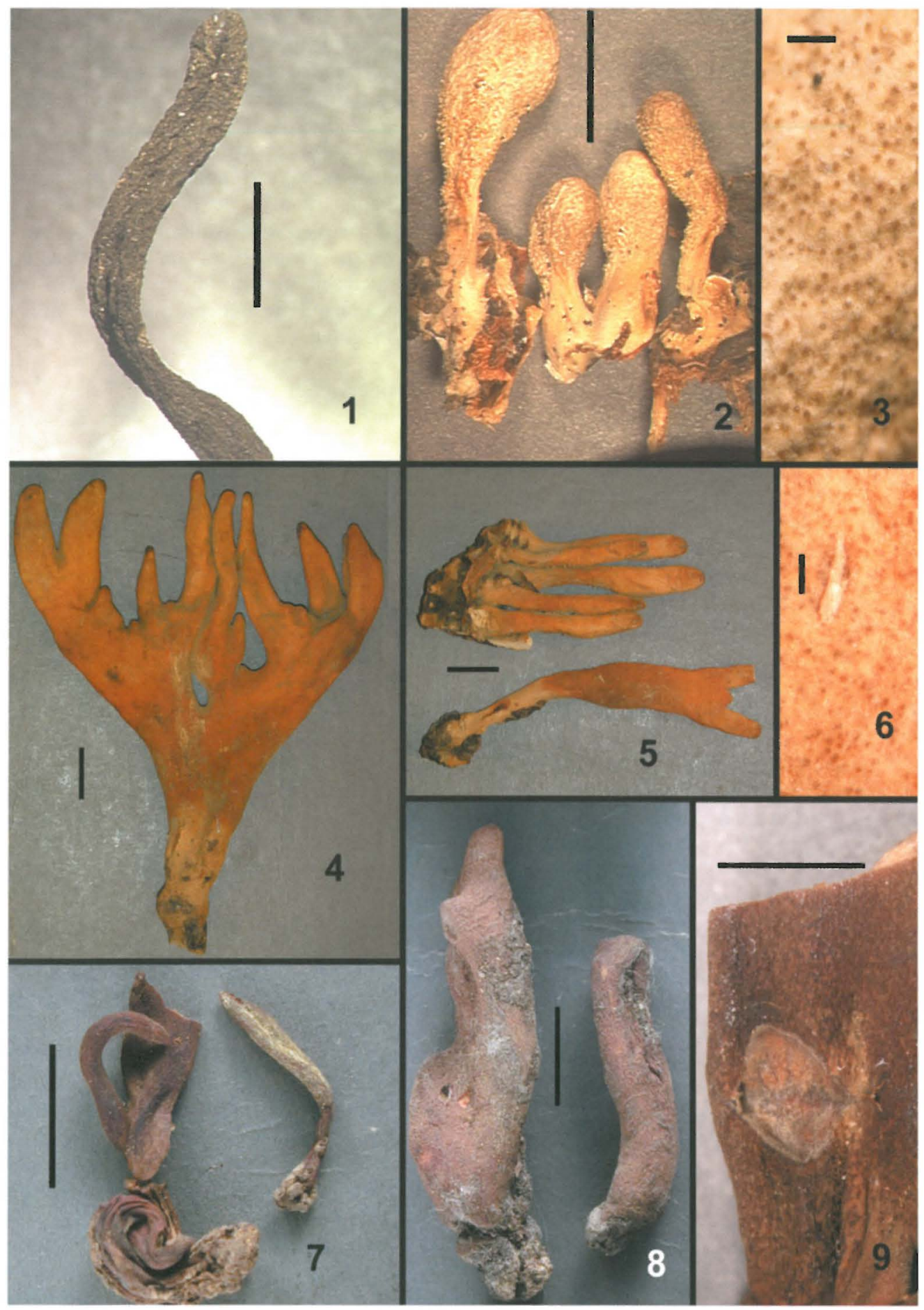

Figs. 1-9. Stipitate stromata of Hypocrea species. -1 . H. africana, from type specimen. Bar $=5 \mathrm{~mm} .-2,3 . H$. alutacea. 2 from Rogerson 86-35, 3 from Spatafora 00-403b. Bar = 0.5 mm. - 4-6. H. cornu-damae, from type. Bars: $4,5=1 \mathrm{~cm}, 6=0.5 \mathrm{~mm} .-7-9$. H. grossa from BPI 745647. Bars: 7, $8=1 \mathrm{~cm} ; 9=1 \mathrm{~mm}$. 


\section{Descriptions of species}

\section{Hypocrea africana (Boedijn) H. Chamb., comb.} nov.

Figs. 1, 10-19.

Basionym: Podostroma africanum Boedijn, Ann. Mycol. 36: 314. 1938.

Anamorph. Unknown.

Stromata simple or dichotomously branched, long-cylindrical, with more or less developed, sterile basal part, sometimes slightly attenuated, not different from rest of stroma; stroma $3-15 \mathrm{~cm}$ tall, $0.15-0.75 \mathrm{~cm}$ wide. Fertile part finely warted with protruding perithecial papillae, thus appearing undulating, greyish-green to dull green when young to olive brown to black when mature (30 E6 to $4 \mathrm{~F} 6$ ), $\mathrm{KOH}-$; consistency of dried material horny. In cross-section stromal tissue dark dirty brown, sometimes nearly black; these cells nearly hyaline, but in center of stroma dirty brown cells present, forming loose network between other cells. Surface cells in surface view of vertically oriented hyphal elements, 3.5-5.0 $\mu \mathrm{m}$ wide; in cross section 25-50 $\mu \mathrm{m}$ wide; surface cells 5.0$7.5 \mu \mathrm{m}$ long $\times 3.5-6.0 \mu \mathrm{m}$ wide; cell walls visibly thickened, $0.5-1.0 \mu \mathrm{m}$ thick. Perithecia subglobose to elliptical, 300-375 $\mu \mathrm{m}$ high $\times 145-200 \mathrm{~mm}$ wide; perithecial wall $6.5-8.0 \mu \mathrm{m}$ wide; ostiolar canal 55-100 $\mu \mathrm{m}$ long $\times 35-50 \mu \mathrm{m}$ wide; perithecial papilla of chains of thick-walled, pigmented cells; ostiolar canal periphysate. Tissue below perithecia of loosely arranged hyphal elements 5-10 $\mu \mathrm{m}$ long $\times 3.5-6.5 \mu \mathrm{m}$ wide. Asci cylindrical, (118-) $125-150(-163) \times(4.0-) 4.7-6.0(-6.5) \mu \mathrm{m}(\mathrm{n}=14)$, apex conspicuously thickened; ascospores disarticulating into 16, uniseriate part-spores. Partascospores hyaline, heavily verrucose, noticeably tuberculate at top and bottom of each spore pair, dimorphic. Distal part-ascospores cuneate and subacute at tip to ellipsoidal, (6.0-) 6.2-7.2($7.7) \times 4-5 \mu \mathrm{m}(\mathrm{n}=30)$, proximal part-ascospores cuneate, oblong to narrowly ellipsoidal, (6.5-)7.08.2(-9.0) ×3.7-4.5(-5.0) $\mu \mathrm{m}(\mathrm{n}=30)$.

Habitat. On ground, possibly associated with termite runs.

Known distribution. Sierra Leone, known only from a single collection.

HOLOTYPE. Sierra Leone. Njala, associated with termites in forest, 18 Nov 1935 F.C. Deighton M882 (IMI 43922!).

Notes. The fresh stromata of this species are described as "...chrome yellow when young, becoming olive-brown and finally almost black with olive base..." (Boedijn 1938). The several stromata that make up the type specimen are almost black. The thick-walled cells of the stromatal surface and the protruding perithecial papilla of chains of thick-walled cells are distinctive. This unusual anatomy is also found in $H$. nigrovirens Chaverri $\&$ Samuels (Chaverri et al. 2001). This species is also characterized by the presence of pigmented cells interwoven with hyaline cells in the internal tissue of the stroma below the perithecia. These pigmented cells give the internal tissue a discolored appearance. The ascospores are large in Hypocrea africana.

2. Hypocrea alutacea (Pers. : Fr.) Tul. \& C. Tul., Sel. Fung. Carp. 1: 63. 1861. Figs. 2, 3, 20-32. = Sphaeria alutacea Pers. : Fr., Commentatio de fungis clavaeformibus: 12. 1797 : Syst. Mycol. 2: 325.1823 .

= Cordiceps alutaceus (Pers. : Fr.) Link, Handbuch 3: 347. 1833, 'Cordiceps'.

= Claviceps alutacea (Pers. : Fr.) Bail, Nova Acta Acad. Caes. Leop. 29: 22. 1861.

= Fracidia alutacea (Pers. : Fr.) Fr., Bot. Zeit. (Berlin) 22: 189. 1864.

$=$ Podocrea alutacea (Pers. : Fr.) Lindau in Engler \& Prantl, Natürl. Pflanzenfam. 1(1): 364. 1897.

= Podostroma alutaceum (Pers. : Fr.) G.F. Atk., Bot. Gaz. 40: 401. 1905.

= Clavaria simplex Schmidel, Icon. Pl. (ed. Keller): 18.1763 ('1762') p. p. according to Tulasne \& C. Tulasne (1865).

= Sphaeria clavata Sowerby, Col. Fig. Engl. Fung. 2: 159.1799.

= Sphaeria alutacea $\beta$ turgida Fr., Syst. Mycol. 2:325. 1823.

Anamorph. Trichoderma sp.

Stromata clavate, typically gregarious, single or sometimes fused, sometimes appearing to be branched or lobed, fully to mostly fertile. Total stroma length (0.5-)2.5-4.5(-8.0) cm ( $\mathrm{n}=28)$, delineation between fertile and sterile parts sometimes evident. Fertile portion $1.4-3 \mathrm{~cm}$ long $(\mathrm{n}=7)$ $\times 0.5-1.8 \mathrm{~cm}$ wide $(\mathrm{n}=29)$; $\mathrm{KOH}-$, golden yellow, coffee to linoleum brown (5 B6-F7), surface glabrous, sometimes wrinkled, perithecial elevations not evident or surface slightly tuberculate, ostiolar openings visible as small dark brown dots against a pale coffee background. Sterile stipe white to beige, velvety or not, $0.5-2.0 \mathrm{~cm}$ long $\times$ $0.5-1.0 \mathrm{~cm}$ wide $(\mathrm{n}=7)$; adjacent stipes often appearing to have fused, with fissures demarcating 


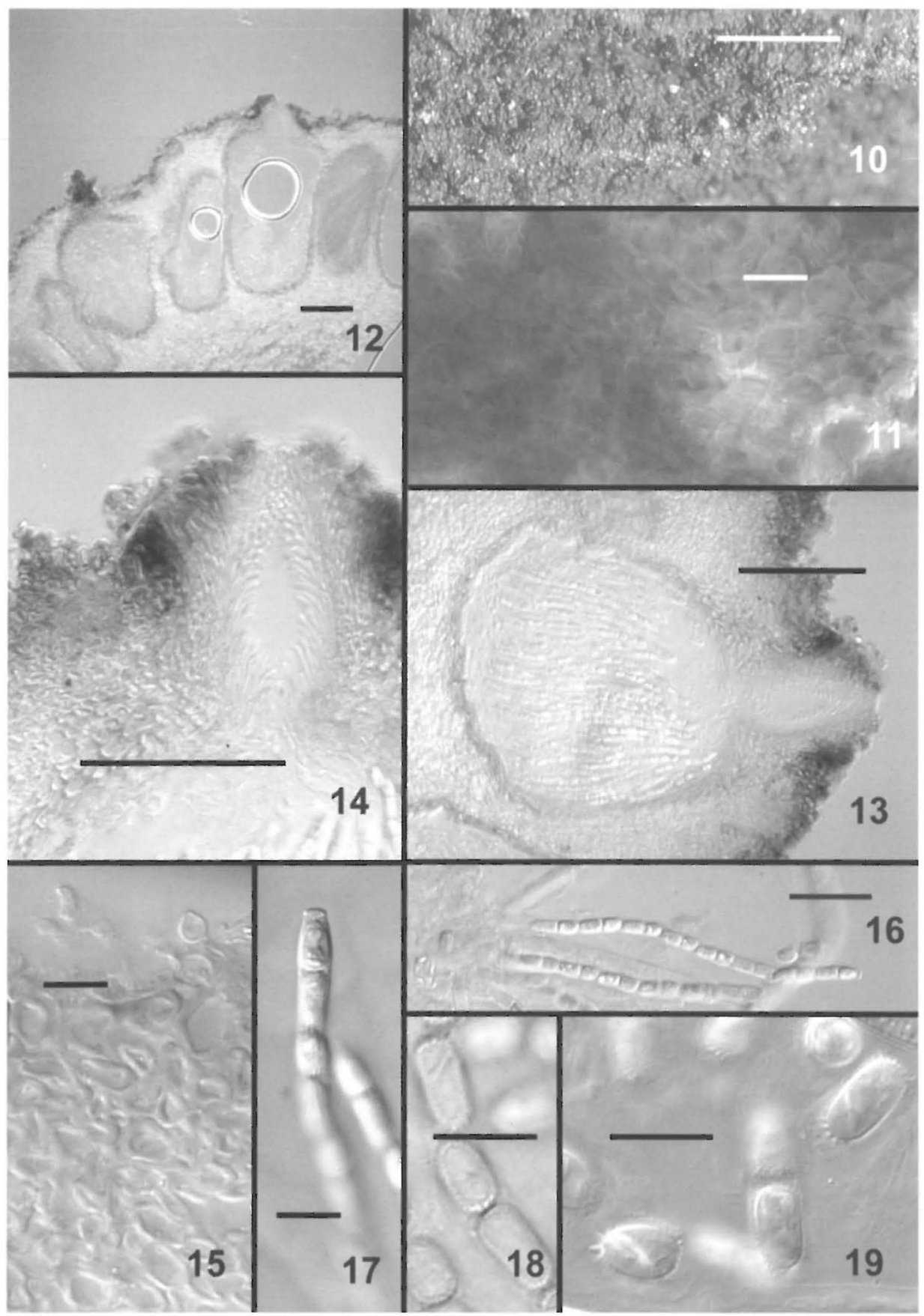

Figs. 10-19. Hypocrea africana, from type. - 10. Stroma surface seen with stereo microscope. Bar $=0.5 \mu \mathrm{m} .-11$. Pseudoparenchymatous cells at stroma surface. Bar $=10 \mu \mathrm{m} .-12,13$. Median, longitudinal section through a perithecium embedded in the stroma. Bars: $12=100 \mu \mathrm{m}, 13=50 \mu \mathrm{m} .-14$. Median longitudinal section through the perithecial papilla showing dark, thick-walled cells. Bar $=50 \mu \mathrm{m} .-15$. Thick-walled, pseudoparenchyma of the interior of the stroma below perithecia. $\mathrm{Bar}=10 \mu \mathrm{m}$. -16 . Ascus with ascospores. $\mathrm{Bar}=20 \mu \mathrm{m}$. -17 . Ascus with thickened apex pierced by a pore. Bar $=10 \mu \mathrm{m}$. -18 . Ascospores in an ascus. Bar $=10 \mu \mathrm{m}$. -19 . Discharged ascospores. Bar $=10 \mu \mathrm{m}$. 


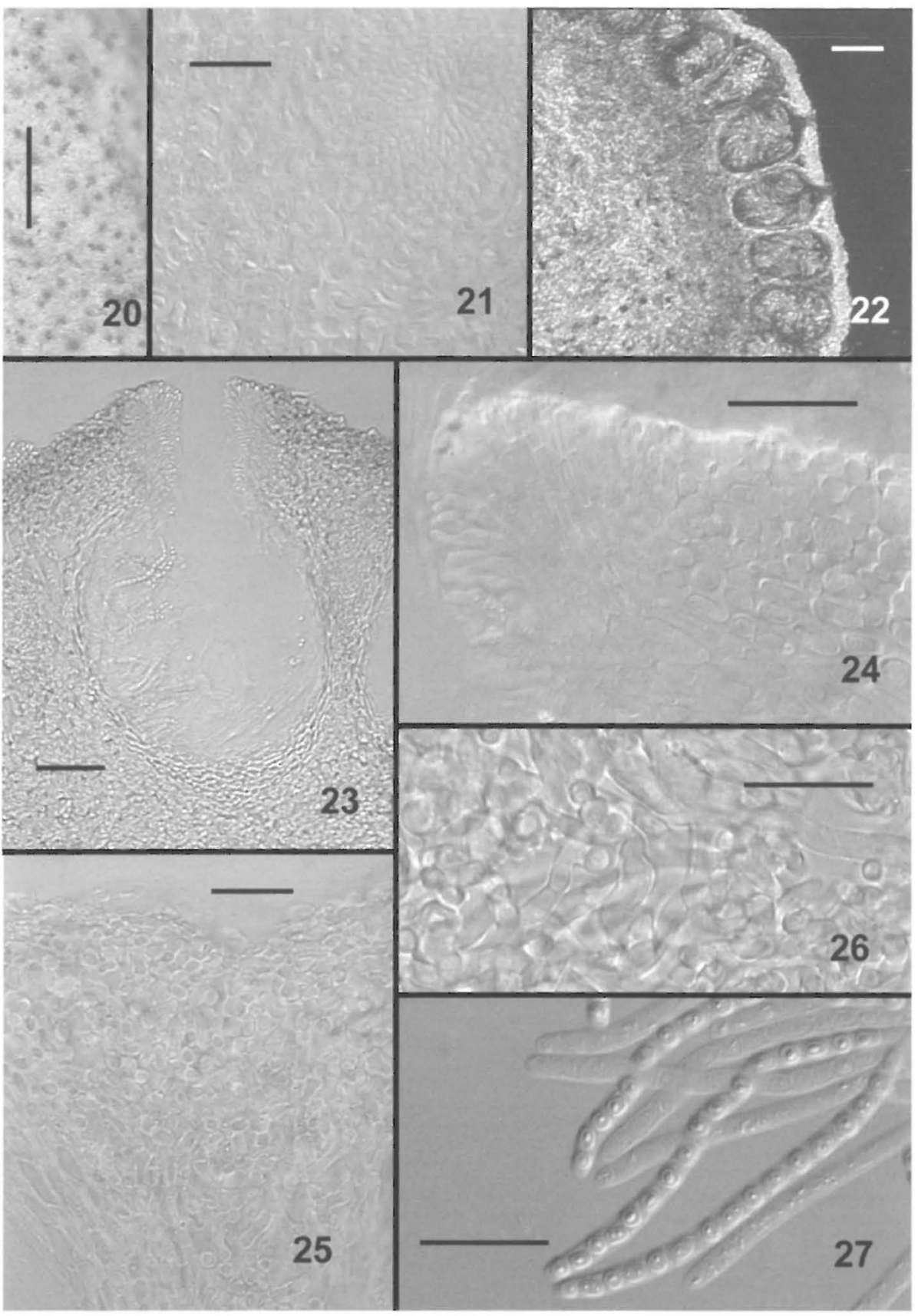

Figs. 20-27. Hypocrea alutacea. - 20. Stroma surface seen with stereo microscope. From Rogerson 86-35. Bar $=0.5$ mm. - 21. Pseudoparenchymatous cells at stroma surface. From H. Harmaja 19 Aug 1979. Bar. $=20 \mu \mathrm{m} .-22$. Section through a stroma showing embedded perithecia. From Rogerson 84-36. Bar $=100 \mu$. -23 . Median longitudinal section through a perithecium. From Samuels 86-538. Bar $=20 \mu \mathrm{m} .-24$. Section through perithecial apex showing cells of the papilla and of the stroma surface. From $L$. Siivonen $(H$ 63261). Bar $=20 \mu \mathrm{m}$. -25 . Stroma surface with hyphal cells below. From H. Harmaja 19 Aug 1979. Bar $=20 \mu \mathrm{m} .-26$. Hyphal cells of the stroma interior below perithecia. From Rogerson 86-35. Bar $=20 \mu \mathrm{m} .-27$. Asci with ascospores. From 6 Oct $1968 \mathrm{~V}$. Haikonen. All DIC except 20. Bar $=20 \mu \mathrm{m}$. 

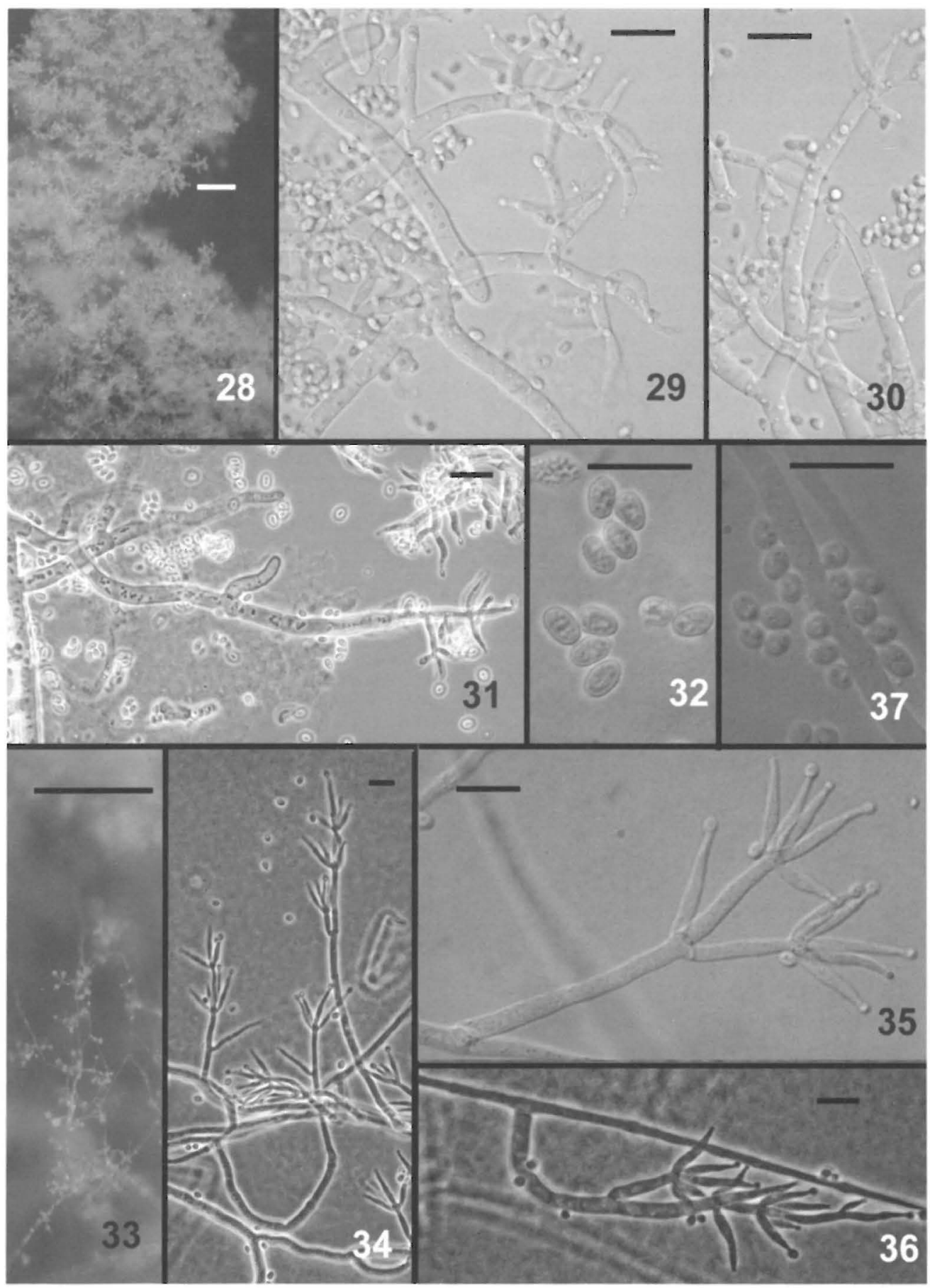

Figs. 28-37. Anamorphs of Hypocrea alutacea and H. leucopus on CMD. - 28-32. H. alutacea, from CBS 332.69. - 28. Conidiophores in aerial mycelium seen with stereo microscope. - 29-31. Conidiophores. - 32. Conidia. All DIC except 28, 31 (PC). Bars $=10 \mu \mathrm{m} .-33-37$. H. leucopus, from DAOM 226070. - 33. Conidiophores in aerial mycelium seen with stereo microscope. - 34-36. Conidiophores. - 37. Conidia. All DIC except 28, 36 (PC). Bars: $33=100 \mathrm{~mm}, 34-37=10 \mu \mathrm{m}$. 
the individual stipes. Cells of stroma surface in surface view elongated and hyphal, 4-17 $\mu \mathrm{m}$ long $\times 3-11 \mu \mathrm{m}$ wide. Stromal surface region pale brown, in section, $28-72 \mu \mathrm{m}$ wide, cells pseudoparenchymatous, $3-8 \times 2.5-6.0 \mu \mathrm{m}$ long, walls $0.1-$ $1.2 \mu \mathrm{m}$ thick. Perithecia crowded below stromal surface, mostly globose to subglobose, $150-400$ $\mu \mathrm{m}$ high $\times 50-287 \mu \mathrm{m}$ wide; perithecial wall 10-35 $\mu \mathrm{m}$ wide; cells at perithecial apex around ostiolar opening, at most, slightly differentiated from stromal tissue, pigmented pale brown; ostiolar canal 30-90 $\mu \mathrm{m}$ long $\times 20-57 \mu \mathrm{m}$ wide, ostiolar canal periphysate. Tissue below perithecia of vertically oriented, thick-walled hyphal cells $4-10 \mu \mathrm{m}$ long $\times$ 2.5-11.0 $\mu \mathrm{m}$ wide, walls $0.5-1.5 \mu \mathrm{m}$ thick, hyaline. Asci cylindrical, (45-)65-90(-113) × (2.2-) 2.5-4.5 $(-5.4) \mu \mathrm{m}$, apex slightly thickened; 16-spored, ascospores uniseriate. Part-ascospores $(n=330)$, hyaline, finely verrucose, dimorphic; distal part globose to subglobose (2.2-)2.7-3.7(-4.5) × (2.2-) $2.5-$ $3.5(-4.5) \mu \mathrm{m}$; proximal part subglobose to conic $(2.2-) 3.0-4.0(-6.0) \times(1.5-) 2.2-2.7(-3.7) \mu \mathrm{m}$.

Culture: On CMD $>2 \mathrm{~cm}$ diam after $6 \mathrm{~d}$; aerial mycelium lacking, diffusing pigment lacking; conidia forming in 2-3 concentric rings. Aggregates cottony with fertile branches protruding, easily removed from agar surface; greyish to almost dark green (28 E6) fading to pistachio green (27 C3 to 28 $\mathrm{C} 4)$ to near white at margin. Colonies on PDA faster than on CMD. On PDA $>3 \mathrm{~cm}$ diam after $6 \mathrm{~d}$; no diffusing pigment formed; conidia formed profusely in dense concentric rings alternating with mycelial production, uniformly cottony, greyish to dull green (28 E6 to $27 \mathrm{C} 3$ ) with age, progressively lighter green toward the margin; noticeably sweet odor. Conidiophores formed on PDA, fertile branches 35-72 mm long ( $\bar{x}=56.92, \mathrm{SD} \pm 14.82, \mathrm{n}=5, \mathrm{R}=36.99)$, less frequently rebranched; phialides arising singly along length of branches and in cruciate whorls of ca. 3 at branch tips. Phialides $(n=30)$ lageniform, tapering uniformly from base to tip, at most only slightly swollen toward middle (7.5-)9.5-13.5(15.7) $\mu \mathrm{m}$ long, at base (1.7-)2.2-3.2(-3.5) $\mu \mathrm{m}$ wide, arising from a cell (1.7-) $3.0-4.0(-4.7) \mu \mathrm{m}$ wide. Conidia oblong to ellipsoidal (2.2-) $2.7-4.5(-5.2) \times$ (1.0-) 1.2-1.7(-2.0) $\mu \mathrm{m}(\mathrm{n}=60)$, lacking a visible basal abscission scar, smooth, green. Chlamydospores not seen.

Habitat. On corticated, but typically wet, rotten wood; also found on wood chips.

Known distribution. Europe, North America.
NEOTYPE designated herein. Illustration in Persoon, C.H. (1800): Observ. Mycol. 2: 66. Tab. I, fig. 2 a-c, reproduced at http://nt.ars-grin.gov/ sbmlweb/OnlineResources/FungiOnline.cfm.

Representative specimens examined: Denmark. Aarhus, vic. Skormollen, Moesgaard forest, on branches of probably Fagus sylvatica, on ground, 30 Aug 1999 C. Lange (BPI 843824); Falster, Sønder Alslev, on rotten wood in beech forest, 16 Sep 1980 A. Pohjola (OULU F 49648). Finland. Etelä-Häme. Hattula, Ilamo, substratum not known, 3 Aug 1981 L. Siivonen (H 63261); Lahti, Mukkula, 61, 02-425, 40, substratum not known, 6 Oct 1968 V. Haikonen $(\mathrm{H})$. Keski-Pohjanmaa. Kälviä, Luoto, 7092:323, on timber, 19 Aug 1979 H. Harmaja (H). Germany. Teutoburger Wald, Beller Holz, on decaying wood (CBS 199.73). Japan. Locality unknown (culture CBS 332.69). Sweden. Södermanland, Överselö par., Tynnelsö Island, W of castle, on decayed wood in mixed forest, 8 Sep 1979 N. Lundqvist 12 (UPS). United States. Maryland. Montgomery Co., Cabin John, on wood, 7 Sep 1919 C.H. Kauffman (BPI 630178). Massachusetts. Chester-Blandford Park, Berkshires, on fallen log, Sep 1978 J. Gailun (NY). Michigan. Iron Co., Upper Peninsula, N Iron River, on rotten wood, 31 Aug 1997 A. D. Parker 102 MI (Parker Herbarium). New York. Cattaraugus County, Allegany State Park, on wood, 15 Sep 1984 R.P. Korf, det. C.T.Rogerson 84-36 (NY); Cortland County, Hoxie Gorge, on log, 1 Oct 1986 A. Methven, det. C.T. Rogerson 86-35 (NY); Hamilton County, Racquette Lake, on wood, 6 Sep 1986 G.J. Samuels \& K.F. Rodrigues, det. G.J. Samuels 86-538 (NY); Warren Co., Lake Sherman, on log, 25 Sep 1969 C.T. Rogerson \& S.J. Smith (NY). North Carolina. Macon Co., Nantahala National Forest, on wood, 8 Sep 1988 K.F. Rodrigues, det. G.J. Samuels 88-60 (NY).

Notes. Hypocrea alutacea is characterized by the following features: (1) occurrence on woody substratum and gregarious habit, (2) dark brown perithecial ostioles contrasting with pale tan to greyish brown stroma and a creamy white sterile stipe, (3) tendency for stromata to become spathulate or branched, and (4) Trichoderma anamorph with green conidia. Another distinguishing character is the thickness of cell walls of the stroma surface and hyphal elements of the inner stroma. The formation of brown pigment in the cells of the ostiolar canal and in the cells of the stroma surface as seen in cross section is unusual in Hypocrea.

The original gathering of Sphaeria alutacea has apparently been lost and no potential neotype specimen exists in Persoon's herbarium (L), thus a neotype of $S$. alutacea is designated as the original illustration of $S$. alutace a by Persoon (1800) examined in the copy at the U.S. National Fungus Collections, BPI. 


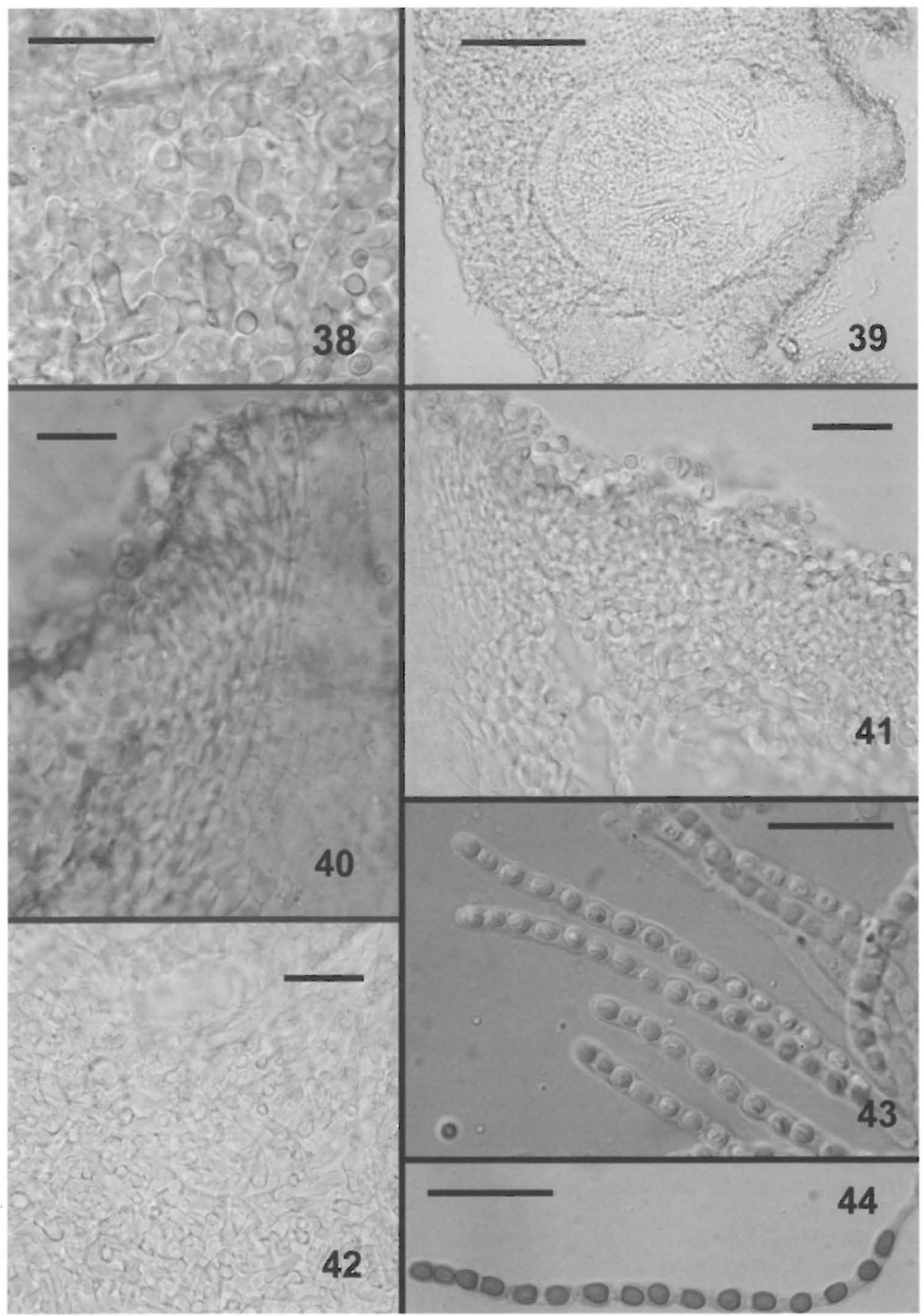

Figs. 38-44. Hypocrea cornu-damae, all from type. - 38. Stroma surface showing hyphae with free ends. - 39. Median longitudinal section of a perithecium embedded in a stroma. - 40. Perithecial papilla. - 41. Perithecial papilla and surface region of stroma showing hyphal region below the surface. -42 . Internal region of stroma below perithecia showing intertwined hyphae. $-43,44$. Asci and ascospores. 44 stained in cotton blue. All DIC. Bars $=20$ $\mu \mathrm{m}$ except $39(=100 \mu \mathrm{m})$. 
3. Hypocrea cornu-damae Pat., Bull. Soc. Mycol. France 11: 198. 1895. Figs. 4-6, 38-44. $=$ Podocrea cornu-damae (Pat.) Lindau in Engler \& Prantl, Natürl. Pflanzenfam. 1(1): 365. 1897. = Podostroma cornu-damae (Pat.) Boedijn, Bull. Jard. Bot. Buitenzorg, ser 3, 13:274. 1934. Anamorph: Unknown.

Stromata simple and cylindrical to narrowly clavate to flabelliform or antler-shaped and dichotomously branched below the tip, 7-9 cm tall, flattened, $0.5-0.7 \mathrm{~cm}$ thick, largest stroma in type collection ca. $1 \mathrm{~cm}$ broad at base, spreading to 8 $\mathrm{cm}$ at top, several cylindrical stromata arising from a common base, buff-orange, stipe sterile; surface glabrous, smooth, perithecial elevations not visible, ostiolar openings appearing as minute orange dots, $\mathrm{KOH}-$. Cells of stroma in surface view appearing as intertwined hyphae with some short, free ends. Surface region of stroma ca. 35 $\mathrm{mm}$ thick in section, of intertwined, 2.5-3.5 $\mu \mathrm{m}$ wide, thin-walled hyphae. Tissue below surface region intertwined hyphae. Perithecia elliptical in section, $280-340 \mu \mathrm{m}$ tall, $130-200 \mu \mathrm{m}$ wide. Tissue below perithecia intertwined, 6-7 $\mu \mathrm{m}$ wide, thin-walled hyphae. Perithecial papilla formed of rows of small, thin-walled cells, papilla barely protruding through stroma surface. Asci cylindrical, $(131-) 135-158(-180) \times(5.0-) 6.5-8.2(-9.8) \mu \mathrm{m}$, apex thickened, with a ring. Part-ascospores hyaline, finely spinulose, dimorphic; distal part subglobose to slightly conical, (3.0-)3.5-4.0(-4.5) $\times$ (2.8-) 3.0-3.2(-4.0) $\mu \mathrm{m}$; proximal part ellipsoidal to cuneate or subglobose, (3.0-)3.2-4.0(-5.0) $\times$ (2.3-)2.5-3.2(-4.0) $\mu \mathrm{m}$.

Habitat. Growing on rotten wood.

Known distribution. Reported from Japan (Doi 1967) but confirmed only for type collection from China (Tibet).

HOLOTYPE. China. Tibet. Su-tschuen (date and collector not known) (FH!).

Notes. Boedijn (1934) reported that the ascospores of this species are smooth. The ascospores in the type collection are very finely spinulose, thus could have appeared smooth to Boedijn. Doi (1967) reported a Trichoderma anamorph for this species but the description given by Doi for that teleomorph suggests $H$. grossa rather than $H$. cornu-damae.

\section{Hypocrea daisenensis (Yoshim. Doi \& Uchiy.) H. Chamb., comb. nov. \\ Basionym: Podostroma daisenense Yoshim. Doi}

\& Uchiy., Bull. Nat. Sci. Mus. Tokyo 1, ser. B, 13: 129. 1987.

Anamorph. Unknown.

Habitat. Growing on ground, possibly from dead, underground wood.

Known distribution. Known only from type locality.

HOLOTYPE. Japan. Tottori Pref., Saihaku-gun, Mt. Houki-Daisen, Masumizugahara, 4 Sep 1974 S. Uchiyama 168 (TNS-F-243748, not available for examination).

Notes. In the protologue, Doi and Uchiyama (1987) state that $H$. daisenensis resembles $H$. africana in the shape and size of stroma, and that the part-spores are generally "pusticulate" but tuberculate at the poles. The stromata of $\mathrm{H}$. daisenensis are cream-carnose, while those of $H$. africana become almost black at maturity. Doi \& Uchiyama (1987) stated that the perithecia and hyphae of stromal tissue are larger in $H$. africana than in $H$. daisenensis. In addition, the part-ascospores described for $H$. daisenensis (distal part 2.7-3.3 $\times 2.3-3.1 \mu \mathrm{m}$, proximal part 3.0-4.1 $\times 2.2$ $2.9 \mu \mathrm{m}$ ) are much smaller than those of $H$. africa$n a$, which are always larger than $4 \mu \mathrm{m}$.

5. Hypocrea grossa Berk. in Hooker's J. Bot. and Kew Gard. Misc. 3:206. 1851. Figs. 7-9, 45-51. $=$ Podocrea grossa (Berk.) Lloyd, Mycol. Writ. 7: 1259. 1924.

= Podostroma grossum (Berk.) Boedijn, Bull. Jard. Bot. Buitenzorg, ser. 3, 13: 273. 1934.

Anamorph. Possibly Trichoderma.

Stroma branched or unbranched, flattened or elliptical in section, cylindrical to more or less flabelliform or dichotomously branched, sometimes several arising from a single point, variable in stature, $3.0-17.0 \mathrm{~cm}$ tall, $0.5-1.5 \mathrm{~cm}$ wide, $0.5-0.7 \mathrm{~cm}$ thick, yellowish-brown, reddish brown to violet brown (9 E8 to $10 \mathrm{E} 7$ ), often nearly black when dry, $\mathrm{KOH}-$, ostiola barely visible as minute, dark dots. Surface of stroma plane, longitudinally wrinkled or furrowed (possibly from drying). Cells at surface of stroma in face view pseudoparenchymatous, 5-20 $\mu \mathrm{m}$ diam, thin-walled. Surface region of stroma ca. $35 \mu \mathrm{m}$ wide, cells angular to subglobose, $5-10 \times 2-8 \mu \mathrm{m}$, thin-walled, walls dark orange. Tissue below surface loosely intertwined, hyaline, thin-walled hyphae. Perithecia mostly subglobose, (226-)245-280(-300) $\mu \mathrm{m}$ tall, (110-)125$185(-250) \mu \mathrm{m}$ wide; cells of perithecial papilla protruding through stroma surface, clavate; ostiolar 


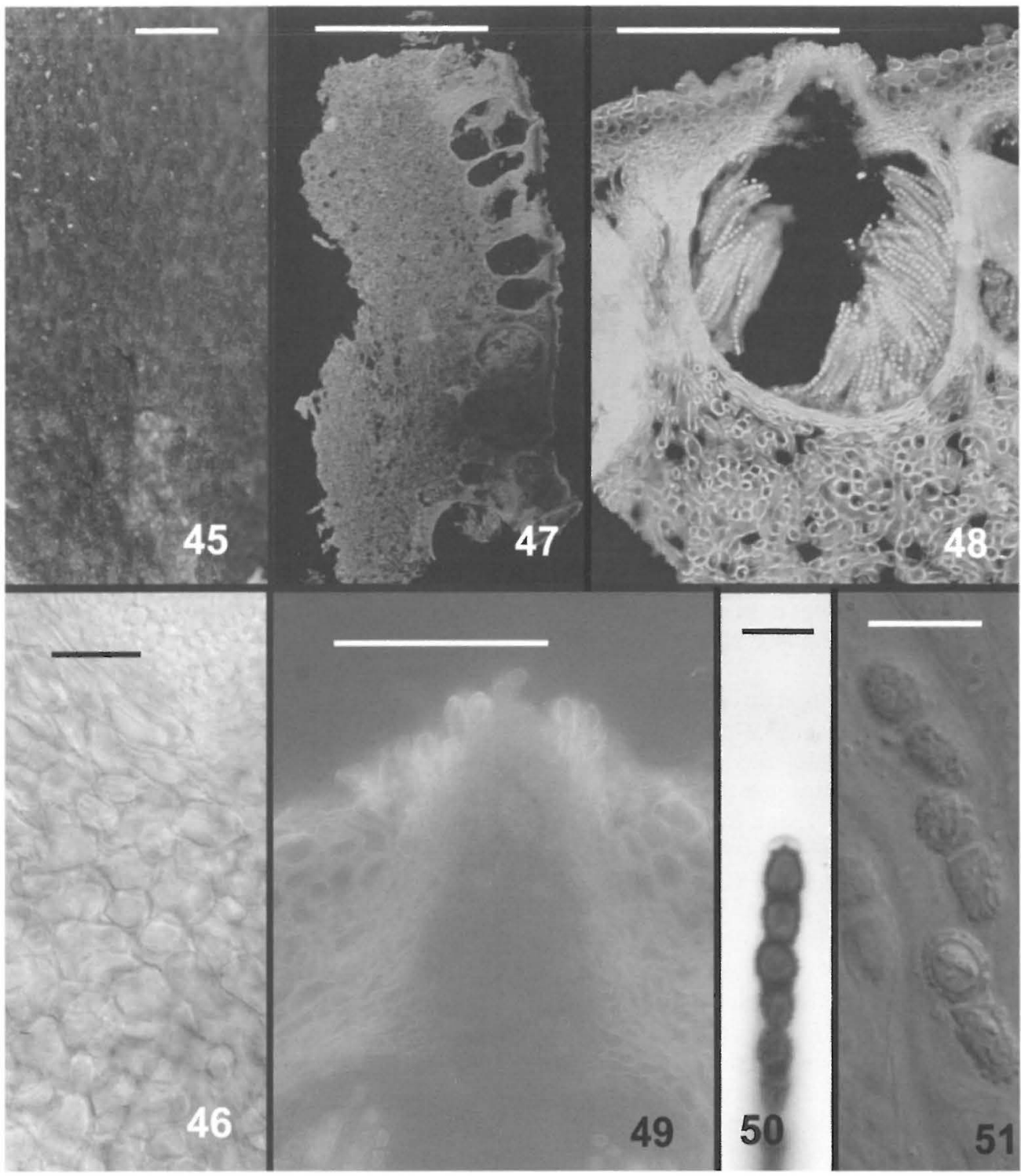

45-51. Hypocrea grossa, all from BPI 745647 except 51 (OULU F 30071). - 45. Surface of stroma seem in stereo microscope. Bar $=1 \mathrm{~mm}$. -46 . Surface of the stroma showing pseudoparenchymatous cells. DIC. Bar $=20 \mu \mathrm{m} .-47$. Section through the outer region of a stroma showing perithecia. FL. Bar $=0.5 \mathrm{~mm} .-48$. Median longitudinal section through a perithecium. FL. Bar $=100 \mu \mathrm{m} .-49$. Perithecial papilla. PC. Bar $=50 \mu \mathrm{m} .-50$. Ascus apex, thickened and with a pore. Cotton blue. DIC. Bar $=1 \mu \mathrm{m} .-51$. Warted ascospores. DIC. Bar $=10 \mu \mathrm{m}$.

canal 45-75 $\mu \mathrm{m}$ long, periphysate. Tissue below perithecia of compact, thin-walled hyphae. Asci cylindrical, (43-) 55-75(-82) × (2.5-) 3.0-4.0(-4.5) $\mu \mathrm{m}$, ascospores filling entire ascus, apex thickened, with a ring. Part-ascospores hyaline to yellow (when discharged), heavily tuberculate as seen in cotton blue, flattened at point of disarticulation; distal part-spores subglobose to conical, (2.7-)4.0- 
$6.7(8.0) \times(2.7-) 4.0-5.5(-6.0) \mu \mathrm{m}$; proximal partspores conical to ellipsoidal or cuneate, (3.0-)4.2$6.5(-9.0) \times(2.5-) 3.7-4.7(-5.7) \mu \mathrm{m}$.

Habitat. On ground.

Known distribution. India, Japan, Thailand.

HOLOTYPE. India. Sikkim, Darjeeling, July (date, collector not known) no. 99 (K!).

Additional specimens examined. Japan. Tottori Pref. Saihaku-Gun, Motodani, Mt. Houki-Daisen, near a large, decayed stump of Quercus mongolica, 5 Oct 1970 Y. Doi D.876 (OULU F 30071 ex TNS-F-223564). Thailand. Saraburi Prov. Khao Yai National Forest, on buried wood, 8 Aug 1997 P. Chaverri (Samuels 8342) (BPI 745674).

Notes. The description given here is a composite of the specimens cited above and the description provided by Boedijn (1934). The measurements of perithecia, asci and ascospores are taken from the cited specimens. The type specimen of H. grossa comprises two blackened stromata glued to a piece of stiff paper. No perithecia were seen in either stroma.

Boedijn (1934) reported this fungus to be highly variable in size and shape, fleshy, mostly without distinct stipe. He illustrated unbranched, lanceolate to cylindrical stromata and dichotomously branched stromata arising from a single base, sometimes with a broadened apex bearing a number of short branches. Stromata were described as $3.5-18 \mathrm{~cm}$ long, $0.5-1.5 \mathrm{~cm}$ broad, side branches $0.4-0.7 \mathrm{~cm}$ broad, flattened apex $1-2 \mathrm{~cm}$ broad; vivid to dark red. He also illustrated the ascospores as being conspicuously warted. The concept of $H$. grossa that we accept here is possibly too broad. There are considerable differences in stroma stature and in ascospore sizes.

In size, degree of branching and terricolous habit $H$. grossa strongly suggests $H$. cornudamae, which is also known from Asia. However, significant differences in stromal anatomy and ascospore morphology separate the two species. The surface of the stroma of $H$. grossa (as represented by BPI 745674) is formed of conspicuous, pseudoparenchymatous cells and the perithecial papilla is formed of clavate cells; the ascospores are obviously warted. The surface of the stroma in the type specimen of $H$. cornu-damae is formed of intertwined hyphae and the perithecial papilla is formed of parallel rows of small cells; the ascospores are finely spinulose and smaller than in $H$. grossa. The stroma of $H$. grossa is a shade of red while the stroma of $H$. cornu-damae is yellowish.

Doi (1967) reported a Trichoderma anamorph with green conidia for $H$. cornu-damae (as $P$. cornu-damae). However, the illustrations of stromatal anatomy and ascospores strongly suggest that he had $H$. grossa. We have not examined a specimen from which he derived the anamorph, but the Japanese collection cited above as $H$. grossa was identified by Doi as $H$. cornu-damae.

Boedijn (1934) synonymized Podocrea cordyceps with H. grossa. However, Doi (1967) reported $P$. cordyceps to be on fallen leaves of Fagus crenata suggesting a host preference or endophytic relationship as for $H$. eperuae, whereas $H$. grossa is known from the ground, not associated with a specific plant host.

6. Hypocrea leucopus (P. Karst.) H. Chamb., comb. nov. Figs. 33-37, 52-56, 61-67. Basionym: Podostroma leucopus P. Karst., Hedwigia 31: 294. 1892.

= Sphaeria alutacea Pers. : Fr. $\beta$ albicans Pers., Syn. Meth. Fung. 2: 2. 1801.

= Hypocrea lloydii Bres. in Lloyd, Mycol. Notes 9(176): 87. 1902.

Anamorph. Trichoderma sp. (Hypocreanum group) verticillium-like.

Stromata clavate, single, sometimes fused or branching; stroma separated into fertile and sterile parts by marked attenuated stipe, sometimes slightly confluent with stipe. Total stroma length $(1.5-) 3.0-5.0(-8.0) \mathrm{cm}$ tall $(\mathrm{n}=23), \mathrm{KOH}-$. Fertile part pale yellow to golden brown (4 A4-B8) to (5 B6-D7), KOH-, surface glabrous, sometimes wrinkled, slightly tuberculate from perithecial elevations, ostiolar openings visible as small viscid dots slightly darker than background. Sterile part white to beige, slightly velvety or not, $(0.5-)$ $1.5-3.7(-5.0) \mathrm{cm}$ long $(\mathrm{n}=18) \times 1.0-1.5 \mathrm{~cm}$ diam $(n=16)$, adjacent stipes often fused, with fissures demarcating individual stipes, $\mathrm{KOH}-$. Cells of stromal surface in surface view pseudoparenchymatous, (4-)5-11(-18) $\mu \mathrm{m}$ long $\times(2.0-) 3.5-6.5(-7.5)$ $\mu \mathrm{m}$ wide . Stromal surface in section $25-45 \mu \mathrm{m}$ wide, cells pseudoparenchymatous, $2-11 \mu \mathrm{m}$ long $\times 2-10 \mu \mathrm{m}$ wide, walls $0.2-0.5 \mu \mathrm{m}$ thick, pale yellow. Perithecia crowded below stromal surface, mostly subglobose to elliptic, $137-330 \mu \mathrm{m}$ long $\times$ $57-255 \mu \mathrm{m}$ wide $(\mathrm{n}=241)$; perithecial wall $14-27$ $\mu \mathrm{m}$ wide; ostiolar canal 30-90 $\mu \mathrm{m}$ long; cells at perithecial apex around ostiolar opening small, 
clavate, thin-walled; periphysate. Tissue below perithecia of loosely intertwined hyphal elements, (3-)4-7(-11) um wide, hyphal, nodose elements lacking. Asci cylindrical, (62-)70-90(-114) ×(2.7-) $3.7-4.7(-6.7) \mu \mathrm{m}$, apex slightly thickened, with a pore; 8 -spored, ascospores uniseriate, often with overlapping ends, completely filling each ascus. Part-ascospores $(n=180)$ hyaline, finely spinulose, dimorphic; distal part subglobose to conic, (2.0-) 2.5-3.0(-4.0) $\mu \mathrm{m}$ diam; proximal part cuneate to ellipsoidal $(2.0-) 3.0-4.0(-5.0) \times(1.7-) 2.0-3.0(-3.5)$ $\mu \mathrm{m}$.

Culture. Colonies grown on CMD at $20 \mathrm{C}$ for $14 \mathrm{~d}$ under $12 \mathrm{~h}$ cool white fluorescent light/12 h darkness $60 \mathrm{~mm}$; aerial mycelium scant; lacking a distinctive odor, diffusing pigment lacking; conidiophores arising directly from surface of agar or from aeral mycelium, forming in a single broad continuous band around margin or in 2-3 concentric rings; conidia held in a drop of hyaline liquid at tip of each phialide. Colonies on PDA faster than on CMD, ca $60 \mathrm{~mm}$ diam after $7 \mathrm{~d}$ at 25-30 C in darkness, greyish or canary yellow (1 B6 to 2 B6), when dried, to dull yellow (3 B3) when fresh diffusing pigment produced; conidia formed profusely in dense concentric rings alternating with mycelial production, uniformly cottony; pale, sun, or pastel yellow (1 A3-4 or 2 A4-5) conidia formed in aggregates on rings; distinct odor. Conidiophores $(\mathrm{n}=24)$ formed on CMD (55-)70130(-200) $\mu \mathrm{m}$ long, (2.5-)3.5-6.0(-7.5) $\mu \mathrm{m}$ wide at base, straight, smooth, uniformly thin-walled or sometimes wall conspicuously thickened toward base, sparingly branched over upper half, branches arising at angles of ca. $45^{\circ}$, ca $15 \mu \mathrm{m}$ long $\times 2-$ $4 \mathrm{~mm}$ wide, cylindrical, each terminating in a verticil of 3-5 phialides or phialides arising directly from conidiophores or from fertile branches. Phialides tapering uniformly from base to tip, 10-17 $\mu \mathrm{m}$ long, 1.7-2.5 $\mu \mathrm{m}$ wide at base, straight. Conidia ellipsoidal, 2.7-3.7(-4.5) × 2.0-2.5(-3.0) $\mu \mathrm{m}$ $(\mathrm{n}=90)$, lacking a visible basal abscission scar, smooth, hyaline. Chlamydospores not seen.

Habitat. On ground among litter, typically in mixed forest type.

Known distribution. Northern Europe, North America.

HOLOTYPE. Finland. Etelä-Häme. Tammela, Syrjä (in larvis), 30 Sep 1892 P.A. Karsten 3247 (H!).

Representative specimens examined. Canada. British Columbia. Queen Charlotte Islands, in old spruce grove,
24 Oct 1998 S.A. Redhead 8201 (DAOM 226147). Locality not known, on soil, S. Redhead 8125 (DAOM 226070, culture and specimen). Denmark. Locality not known, on soil, 23 Jul 2000 C. Lange (BPI 843826, culture A.Y. Rossman 3784); Central Jutland. Vilhola near Sdr. Vissing, in litter of Fagus sylvatica, 2 Oct 1999 F. Jensen (BPI 843825). Estonia. Tartumaa County. Järvselja Forest Reserve, on soil, 19 Sep 2003 M. Vaasma \& K. Põldmaa (TAA 170630, culture G.J. Samuels 03-09). Finland. Uusimaa. Sipoo, Hindsby, forest rich in herbs (Betula, Corylus, Picea, Populus tremula, Oxalis acetosella, Salix caprea), on leaf litter, among mosses, humous soil, Grid $27^{\circ} \mathrm{E}$ 6694:402, 25 Jul 1984 R. Saarenoksa $22084(\mathrm{H})$. Etelä-Häme. Lammi, Hauhiala, substratum unknown, 5 Sep 1984 K. Törmäkangas (H); Tammela, Mustiala, versus Särkjärvi, 8 Sep 1897 $J$. Lindroth (H, Herb. Karsten 3248). Pohjois-Karjala. Värtsilä, Savikko, Grid 27E 69077:6888, elev. 75-85 m, substratum unknown, 3 Sep 1993 H. Väre (OULU F 49644). Keski-Pohjanmaa. Kruunupyy, 8 Sep 1981 R. Storbacka (OULU F 49572). Oulun Pohjanmaa. Kiiminki, Hannus, Grid $27^{\circ} \mathrm{E} 72280: 4470$, elev. $43 \mathrm{~m}$, 4 Sep 1997 E. Ohenoja (OULU F 32112). Sweden. Värmland. Nyed sn, Rudsberg, 7 Oct 1945 K.G. Ridelius (UPS). United States. New York. Delaware Co., Arkville, Aug 1916 W.A. Murrill (NY); St. Lawrence Co., 28 Aug 1988 G. Bills (NY, culture G.J. Samuels 88-25); North Carolina, Swain Co., Indian Creek, 14 Aug 1968 C.T. Rogerson CTR 68-81 (NY). Pennsylvania. Pocono Lake Preserve, Aug 1935 C.B. Stifler (BPI 630175). Tennessee. Sevier Col., LeConte Creek, 12 Aug 1968 C.T. Rogerson (NY); Great Smoky Mts. National Park, Cherokee Orchard, 8 Aug 1934 A.J. Sharp (TENN). Vermont. Bennington, Coleville Rd., 29 Aug 1981 P. Raften, det. C.T. Rogerson 81-92 (NY). Virginia. Giles Co., Mountain Lake, 3 Sep 1936 D. Linder (FH).

Notes. When young, the stromata of H. leucopus are pale yellow to butter yellow, becoming golden brown with time. North American specimens of $H$. leucopus tend to be more golden brown than those found in Europe, but in other characters they are indistinguishable. The color of the stroma, the strong constriction between fertile and sterile parts of the stroma, and its occurrence on ground distinguish this species from H. alutacea and other stipitate species of Hypocrea. Moreover, the respective anamorphs separate $H$. leucopus and $H$. alutacea.

Podostroma leucopus has been overlooked in the literature since Atkinson (1905) synonymized it with P. alutaceum. Although he listed this synonymy with a question mark, succeeding authors have accepted this (Doi 1966, Imai 1932, Rossman et al. 1999). Among specimens previously identified as $P$. aluteaceum and examined for this study, three species of Hypocrea are recognized. Two of the species already have names, specifically $H$. leucopus and $H$. alutacea. The following 


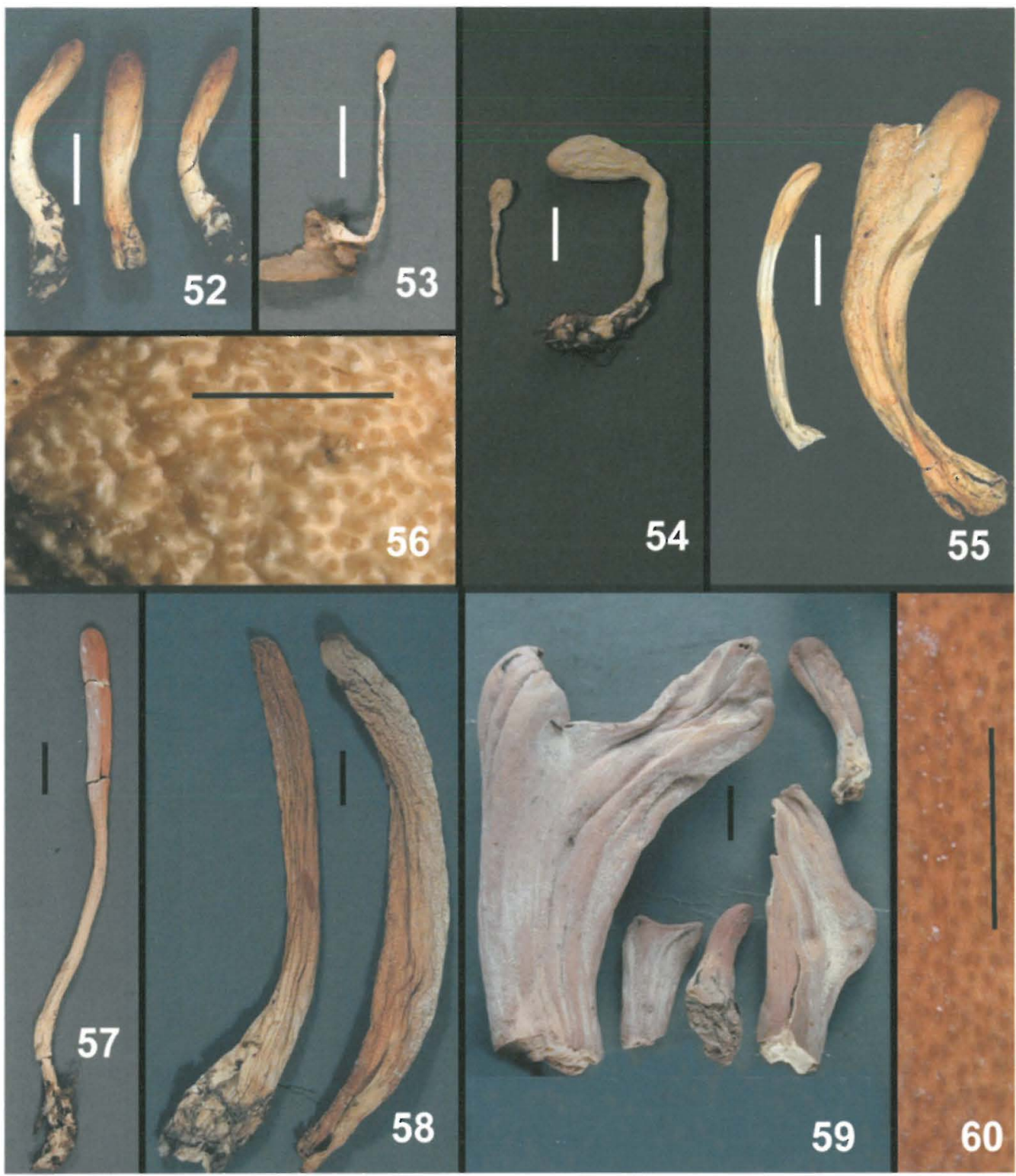

Figs. 52-60. Stipitate stromata of Hypocrea species. - 52-56. H. leucopus. 52 from 5 Sep 1984 K. Törmäkangas; 53 from Saarenoksa 22084; 54 from Jul 2000 C. Lange; 55 from OULU F 49644; 56 from DAOM 226070. - $57-$ 60. H. nybergiana. 57 from OULU F 49597 (type); 58 from Korhonen 4117; 59 from OULU F 49620; 60 from OULU F 49572.

morphological characteristics distinguish these two species: (1) H. leucopus has smaller ascospores than those of $H$. alutacea, (2) the stromatal color of $H$. leucopus tends to be paler and more yellowish than $H$. alutacea, (3) the surface cells and tissue below the perithecia of $\mathrm{H}$. leuco- pus are thin-walled whereas they are thick-walled in H. alutacea, and (4) H. leucopus typically occurs on the ground while $H$. alutacea develops on woody substrata. The third entity is described as a new species, $H$. nybergiana, which is similar to H. leucopus, but is differentiated by the red- 


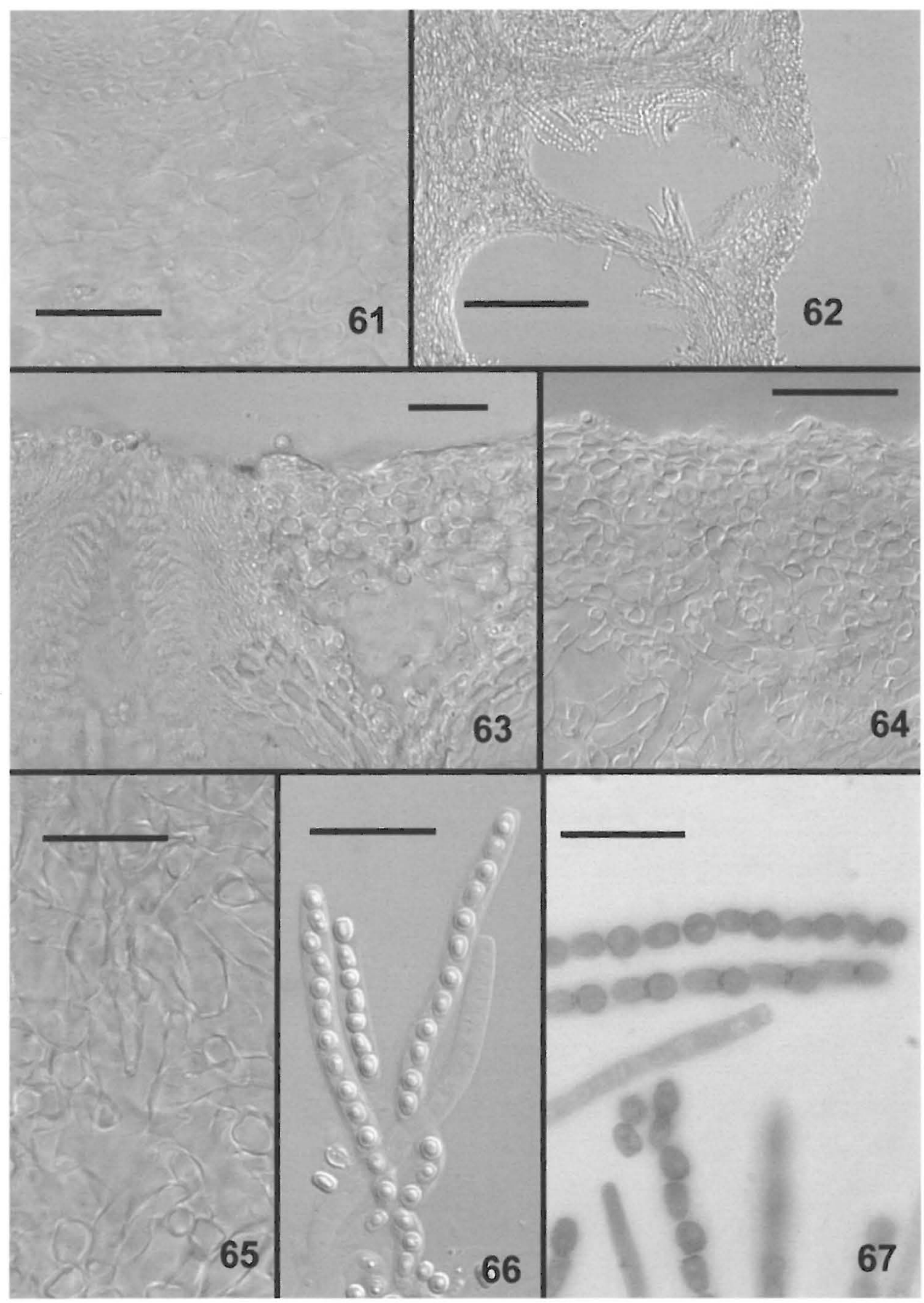

Figs. 61-67. Hypocrea leucopus. - 61. Stroma surface showing pseudoparenchymatous cells. - 62. Median longitudinal section through a perithecium. -63 . Perithecial apex and adjacent stroma surface. -64 . Stroma surface region formed of pseudoparenchyma, loosely disposed hyphae below. -65 . Internal tissue of stroma below perithecia. -66 . Asci. - 67. Ascospores in asci warted. Cotton blue. Figs. 61, 63 from 5 Sep 1984 K. Törmäkangas; 62 from OULU F 32112, 64-67 from OULU F 49644. Bars $=20 \mu \mathrm{m}$ except $63=100 \mu \mathrm{m}$. 


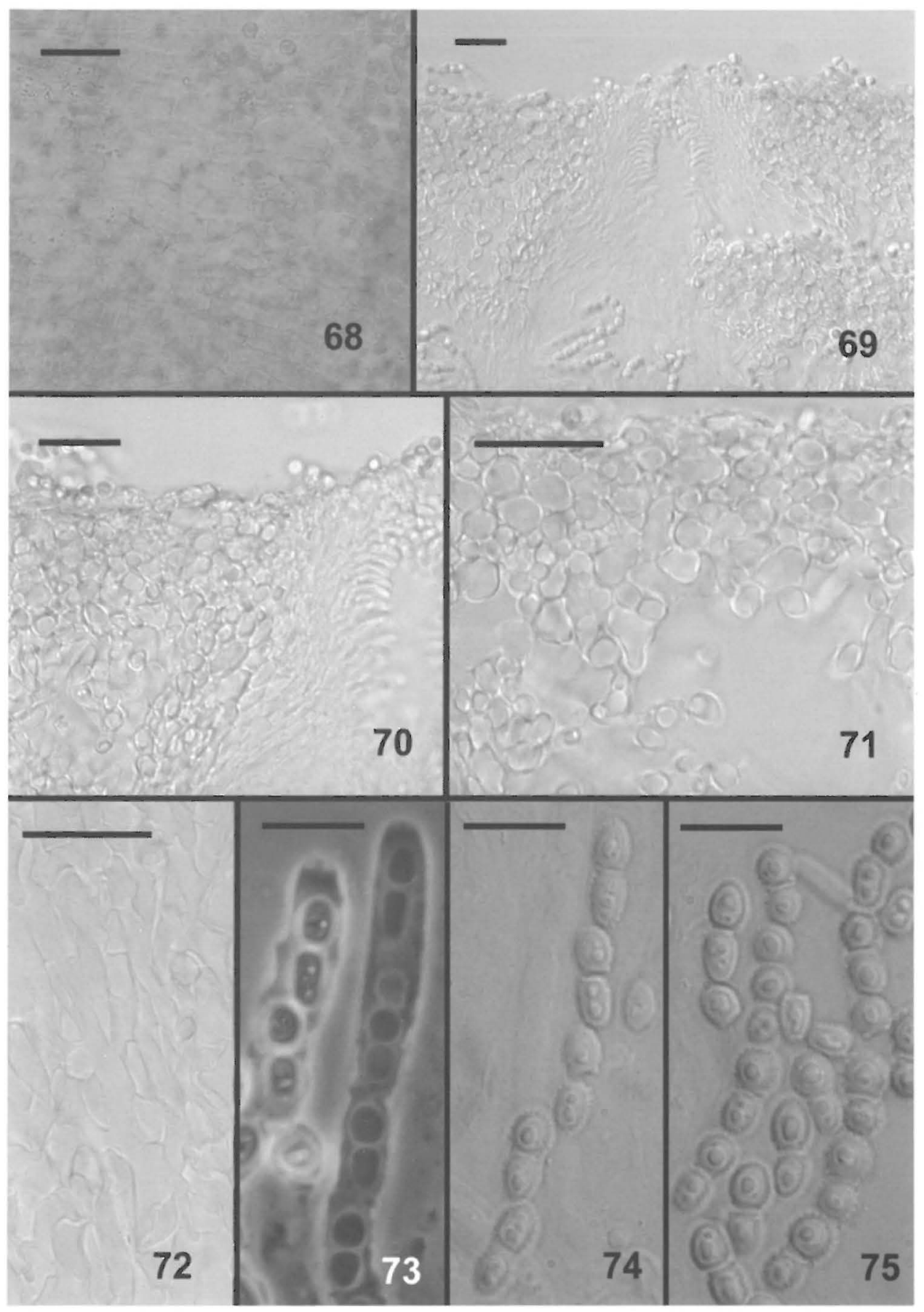

Figs. 68-75. Hypocrea nybergiana. - 68. Stroma surface showing elongated cells. - 69, 70. Section through perithecial papilla and adjacent stroma surface. - 71. Stroma surface showing pseudoparenchymatous cells; loosely disposed hyphae forming below the surface. -72 . Internal tissue of stroma below perithecia. -73 . Ascus apex with apical ring. Cotton blue. PC. Bar $=10 \mu \mathrm{m}$. -74 . Ascus with ascospores. DIC. Bar $=10 \mu \mathrm{m}$. -75 . Discharged ascospores. Bar $=10 \mu \mathrm{m}$. All DIC except $73(\mathrm{PC})$. Bars $=20 \mu \mathrm{m}$ except $73-75=10 \mu \mathrm{m}$. Fig. 68, 73-75 from OULU F 49603; 69-72 from KUO 14577. 
dish-brown to brownish orange stromata and conspicuous scales of rust pigment between the fertile and sterile areas on the stipe.

\section{Hypocrea nybergiana T. Ulvinen \& H. Chamb.,} spec. nov. Figs. 57-60, 68-75. [=Podostroma nybergianum T. Ulvinen, Suursieniopas: 291. 1976, nom. inval., Latin description lacking.]

Stromata cinnamomea vel fulva, clavata. Stipites squamati ad medium, 2.2-15(-22) cm alta, 2.5-4.1 $\mathrm{cm}$ crassa. Perithecia $180-450 \mu \mathrm{m}$ alta, $\times 65-315$ $\mu \mathrm{m}$ lata. Asci cylindrici, $59-100 \times 2.5-5.5 \mu \mathrm{m}$, ad apicem incrassati. Ascosporae bicellulares, hyalinae, minute verrucosae, ad septum disarticulatae; parte distali globosa vel subglobosa, (3.0-) $3.5-4.5(-6.0) \times(3.0-) 3.2-4.0(-4.5) \mu \mathrm{m}$, parte proximi oblonga vel cuneiformi, (3.0-)3.7-5.0(-6.0) $\times$ (2.5-)3.0-3.5(-4.5) $\mu \mathrm{m}$.

HOLOTYPE. Finland. Oulun Pohjanmaa. Haukipudas, Kello, Kalimenkylä, Kalimenoja, 1⁄1/2 $\mathrm{km}$ upstream of Saarela, in spruce forest in mouth of Suo-oja-brook, on Hylocomium-covered ground mixed with spruce needles and twigs, 24 Aug 1967 T. Ulvinen (OULU F 49597, isotype OULUF 49596).

\section{Anamorph. Unknown.}

Stromata narrowly clavate and slender to shallowly branched and robust, $2.2-15(-22) \mathrm{cm}$ long, $0.2-0.9(-7) \mathrm{cm}$ wide at apex, fertile part $1.0-6.5 \mathrm{~cm}$ wide, sterile part $1.5-5.5 \mathrm{~cm}$ long $\times 0.1-0.4 \mathrm{~cm}$ wide, reddish brown to brownish orange, $6 \mathrm{C}-\mathrm{D} 8$; rust-pigmented scales covering median section of stromata, reaching middle of sterile base, stipe beige, $\mathrm{KOH}-$. Stroma surface glabrous, slightly tuberculate from papillate perithecial elevations, ostiolar openings visible as large darker orange dots against slightly lighter colored background; transverse striations sometimes visible in stroma surface. Cells of stromal surface in surface view longitudinally arranged, often rectangular cells, 5.5-11.5 $\mu \mathrm{m}$ long $\times 2.5-8 \mu \mathrm{m}$ wide. In section, stroma surface 30-70 $\mu \mathrm{m}$ wide, cells at surface pseudoparenchymatous, 3-10 $\mu \mathrm{m}$ diam, walls visibly thickened and pigmented orange to rust-colored clearly differentiated from underlying tissue by orange brown or rust pigment. Tissue immediately below surface region of compact to loosely intertwined hyphal elements. Perithecia globose to elliptical, $180-450 \mu \mathrm{m}$ tall $\times 65-315 \mu \mathrm{m}$ wide $(\mathrm{n}=88)$; perithecial wall red-brown in $3 \% \mathrm{KOH}$; ostiolar canal of small, thin-walled cells, ostiolar canal 37-100 $\mu \mathrm{m}$ long, periphysate. Tissue below perithecia of loose hyphal elements vertically oriented, 4.0-8.5 $\mu \mathrm{m}$ long $\times 3.5-11.0 \mu \mathrm{m}$, walls ca. 0.5 $\mu \mathrm{m}$ thick. Asci cylindrical, (63-)75-100(-130) $\times$ (3.2-)4.0-6.2(-7.5) $\mu \mathrm{m}(\mathrm{n}=89)$; ascospores partly biseriate above or uniseriate, filling entire ascus. Part-ascospores hyaline, becoming conspisuously verrucose and slightly thick-walled, dimorphic; distal part-ascospores globose to subglobose, $(3.0-) 3.5-4.5(-6.0) \times(3.0-) 3.2-4.0(-4.5) \mu \mathrm{m}$ $(n=149)$; proximal part-ascospores ellipsoidal to wedge-shaped, (3.0-)3.7-5.0(-6.0) $\times(2.5-) 3.0-$ $3.5(-4.5) \mathrm{mm}(\mathrm{n}=149)$.

Habitat. On ground among forest litter, typically associated with moss.

Known distribution. Northern Europe.

Etymology. Named in honor of Mr. Wolmar Nyberg (1870-1958), a Finnish bank manager and amateur mycologist who made significant contributions to knowledge of Finland's mycobiota.

Representative specimens examined. Finland. PohjoisSavo. Siilinjärvi, ca. $200 \mathrm{~m} \mathrm{NE}$ of N end of Pahkalampi, moist old Picea abies forest, on decaying twigs of Picea abies, Grid 27²E 7009:538, 5 Aug 1974 H. Heikkilä 606 (KUO 14577). Kainuu. Puolanka, Väyrylä, Pääkkö, Iso Vuorlampi, E side, coniferous spruce dominated forest on mossy ground with Geranium, Grid $27^{\circ} \mathrm{E} 7185: 537$, 9 Sep 1986 T. Ulvinen \& H. Väre (OULU F 49603). Oulun Pohjanmaa. Kiiminki, Piikkikoskenkangas, W-margin, mixed spruce forest, Grid $27^{\circ} \mathrm{E} 72262: 4453$, 8 Sep 1973 E. Ohenoja (OULU F 49620). Koillismaa. Kuusamo, Oulanka, Kiutaköngäs, Haaralampi, eutrophic forest, Grid $27^{\circ} \mathrm{E} 7365: 605,30$ Aug $1981 \mathrm{M}$. Korhonen $4117(\mathrm{H})$. Perä-Pohjanmaa. Tervola, Palokivalo, on calcareous ground, 5 Sep 1996 M. Ohenoja \& E. Ohenoja (OULU F 31634). Sweden. Jämtland. Bracke commune, 5 Sep 1997 D. Laber $(\mathrm{H})$.

Notes. In young stages, the stromata are pale brown to brownish orange, becoming more reddish golden with time. Some stromata become very large and fully fertile with scales lacking. This species is differentiated from other stipitate Hypocrea species based on the presence of rustypigmented scales and large stature.

\section{Doubtful species}

Podocrea solmsii (E. Fisch.) Lindau var. corniformis Bres., Ann. Mycol. 5: 241. 1907.

The type specimen was not examined. Based on the type description this taxon is reported to occur on wood and has stromata that are dull yellow with a mixture of grey and brown, appearing 
dark tawny suggesting that it is similar to Hypocrea gigantea. Boedijn (1934) synonymized this name with Podostroma grossum (三 Hypocrea grossa).

Podocrea zeylanica Petch, Ann. Roy. Bot. Gard. Peradeniya 6: 230. 1917.

After examination of the type specimen at K, this species appears to be closely related to $H$. alutacea, if not the same. It shares a similar habitat on wood and the stromata are often clustered, simple, clavate, or cylindric, sometimes compressed, with apex often irregularly lobed, rufous or pale brown with darker ostioles. However, the specimen is in poor condition lacking asci and ascospores and an exact determination was difficult from available material.

\section{Excluded taxa}

Podocrea anomala Lloyd, Mycol. Notes 6 (65): 1053, pl. 180 (f. 1960, 1961). 1921.

Based on the type specimen this fungus is not a member of the Hypocreaceae, rather the stromata resemble those of the Xylariaceae in being darkly colored. Lloyd reported having seen Hypocrea-like spores. Although the asci are immature and contain no ascospores, the ascal apex is blue in Melzer's reagent, a characteristic that is typical of the Xylariaceae and is unknown in the Hypocreaceae.

HOLOTYPE. Philippines. Luzon, Mt. Maquiling, on wood, 22 Sep 1920 E. Collado (BPI 631731).

Podocrea cornu-bovi R. Heim \& T. Herrera, Rev. Mycol. 25:215. 1960.

This species is known only from type description; the type specimen could not be found and is considered lost. The protologue suggests that this fungus is an immature Xylariaceae rather than a member of the Hypocreaceae based on the following: 1) cortex of the perithecia black with a white fertile stroma; 2) stromata having a black, hardened base; and 3) large, ovoid to subglobose, hyaline, non-septate spores, 7-11 × 6-8,5 $\mu \mathrm{m}$, that do not separate into part-spores.
Podostroma solmsii (E. Fish.) Imai f. octosporum Yoshim. Doi, Bull. Nat. Sci. Mus. Tokyo B 4: 24. 1978, 'octospora'. Figs. 76-84. Type material of this forma was not made available to us. A Japanese specimen of $P$. solmsii $\mathrm{f}$. octosporum was examined: Japan. Ishikawa Pref. Kanagawa City, Bessho-cho, Gando-Yama, in bamboo forest, on 'eggs' of Phallus sp., 22 Oct 1978 Y. Ikeda 493-3 (OULU F 30072 ex TNS-F193041). This specimen is not a Hypocrea and may represent an undescribed genus of the Hypocreaceae. The specimen conforms to what was described and illustrated by Doi (1978) for the forma. The ascospores are fusiform to ellipsoidal, unicellular and warted; they do not become septate. On many ascospores there appears to be a blunt apiculus at each end. The perithecia completely cover the surface of a beige stroma, ca 3 $\mathrm{cm}$ tall that arises from the 'egg' of a Phallus species. We cannot state unequivocally that the 'stroma' is not a part of the Phallus covered with perithecia of the parasite. The combined characters of mycoparasitism and fusiform warted, possibly apiculate ascospores suggest that this taxon is derived from Hypomyces.

Podocrea xylarioides Lloyd, Mycol. Notes 5(57): 844, fig. 1412. 1919.

The type specimen has characteristics typical of the Xylariaceae rather than Hypocreaceae. The stromata are darkly colored and hard when dried rather than soft or brittle. Although asci in the specimen at BPI are immature, the apex turns blue in Melzer's reagent.

HOLOTYPE. Japan. Ishikari Prov., 18 Jul 1918, A. Yasuda (BPI 631733).

Acknowledgments: The 'PEET TEAM', including Priscila Chaverri, Sarah Dodd, Barrie Overton, Kadri Põldmaa, and Nancy Wenner are acknowledged for their contributions of unpublished information and support. Christian Lange and Joseph Spatafora provided recent collections of $H$. alutacea and H. leucopus. Many thanks are extended to the numerous herbaria that sent reference and type specimens with major contributions from the following: BPI, CBS, CUP, FH, H, HKAS, HMAS, K, KUO, NY, OSC, OULU, and UPS. Research was supported by a PEET grant from the National Science Foundation (9712308) to The Pennsylvania State University. 


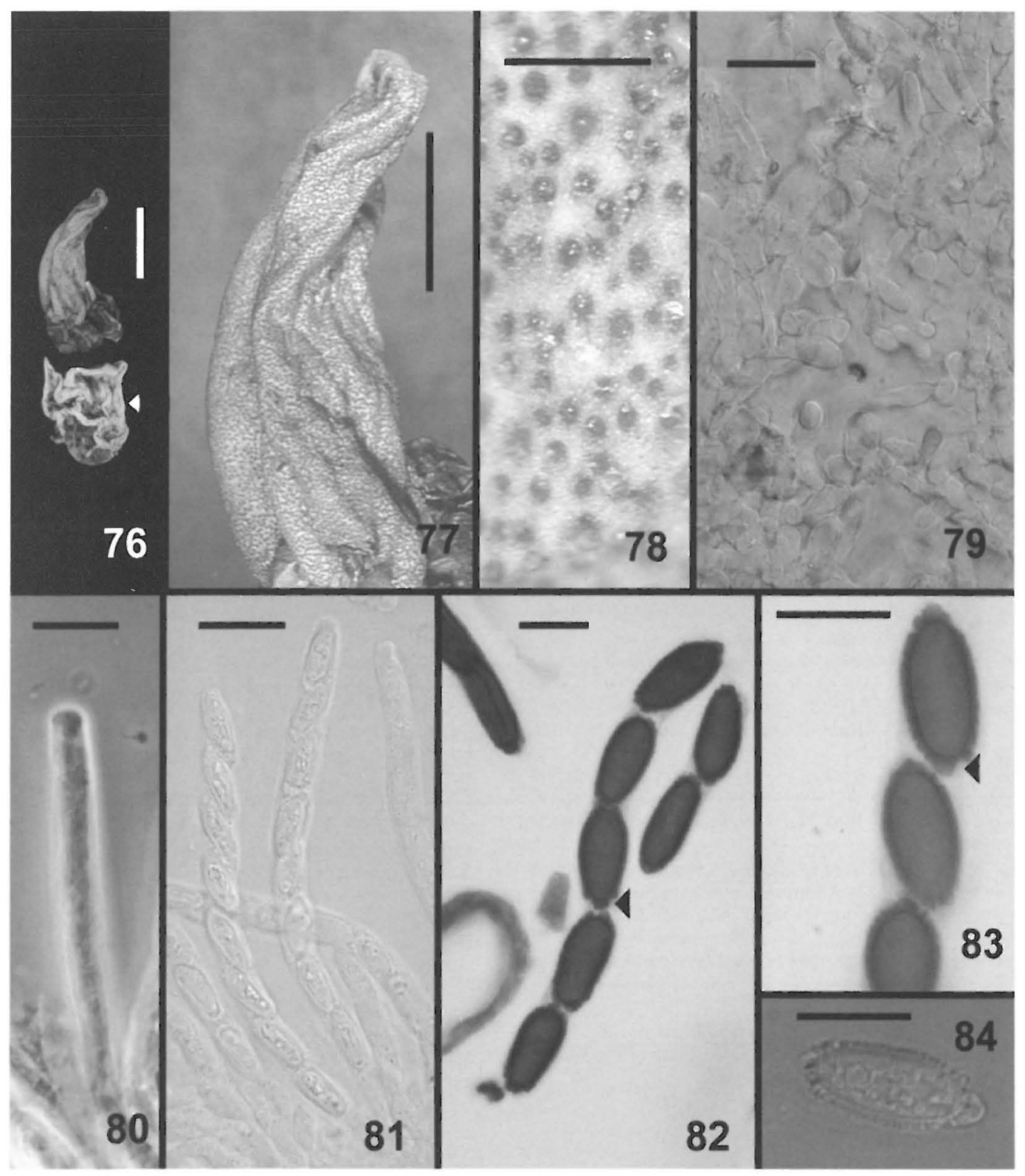

Figs. 76-84. 'Podostroma' solmsii f. octosporum, all from OULU F 30072. - 76. Habit of stroma arising from 'egg' of a Phallus sp. (arrow). Bar $=2 \mathrm{~cm} .-77$. Stroma of Hypocrea. Bar $=1 \mathrm{~cm}$. -78 . Stroma surface as seen in stereo microscope. Bar $=0.5 \mathrm{~mm}$. -79 . Stroma surface, hyphae with free ends. Bar $=20 \mu \mathrm{m} .-80$. Immature ascus with apical ring. Bar $=20 \mu \mathrm{m}$. -81 . Ascus with developing ascospores. Bar $=20 \mu \mathrm{m} .-82,83$. Nearly mature spores in asci. Cotton blue. Arrow shows possible apiculus. -84 . Discharged ascospore. Bar $=10 \mu \mathrm{m}$. Figs. $76-78$ stereo microscope; 79, 81-84 DIC; 80 PC. 


\section{References}

Atkinson, G.F. 1905: Life history of Hypocrea alutacea. - Bot. Gaz. 40: 401-417.

Bissett, J. 1991: A revision of the genus Trichoderma. III. Section Pachybasium. - Canad. J. Bot. 69: 23732417.

Boedijn, K.B. 1934: The genus Podostroma in the Netherlands Indies. - Bull. Jard. Bot. Buitenzorg, Ser. 13, 13: 269-275

Boedijn, K.B. 1938: A new species of the genus Podostroma from Africa. - Ann. Mycol. 36: 314-317.

Chaverri, P., Samuels, G.J., Stewart E.L. \& Umaña, L. 2001: Hypocrea nigrovirens, a new species with a gliocladium-like anamorph. - Mycologia 93: 758-763.

Chaverri, P. \& Samuels, G.J. 2003: Hypocrea/Trichoderma (Ascomycota, Hypocreales, Hypocreaceae): Species with green ascospores. - Stud. Mycol. 48: 1-116.

Clements, F.E. \& Shear, C.L. 1931: The genera of fungi. - H.W. Wilson, New York.

Doi, Y. 1966: A revision of the Hypocreales with cultural observations. I. Some Japanese species of Hypocrea and Podostroma. - Bull. Natl. Sci. Mus. Tokyo 9: 345-357.

Doi, Y. 1967: Revision of the Hypocreales with cultural observations. III. Three species of the genus Podostroma with Trichoderma or Trichoderma-like conidial states. - Trans. Mycol. Soc. Japan 8: 54-60.

Doi, Y. 1973: Revision of the Hypocreales with cultural observations. V. Podostroma giganteum Imai, P. cornu-damae (Pat.) Boedijn and Hypocrea pseudogelatinosa sp. nov. - Rep. Tottori Mycol. Inst. (Japan) 10: 421-427.

Doi, Y. 1975: Revision of the Hypocreales with cultural observations. VIII. Hypocrea peltata (Jungh.) Berk. and its allies. - Bull. Nat. Sci. Mus. Tokyo, ser. B, 1: 121-134.

Doi, Y. 1978: Revision of the Hypocreales with cultural observations. XI. Additional notes on Hypocrea and its allies in Japan (1). - Bull. Nat. Sci. Mus. Tokyo, ser. B, 4: 19-26.

Doi Y. \& Uchiyama, S. 1987: A new Podostroma species from Japan. - Bull. Nat. Sci. Mus. Tokyo, ser. B, 13: 129-132.

Holmgren, P.K., Holmgren, N.H. \& Barnett, L.C. 1990: Index Herbariorum Part I: The Herbaria of the World. - Ed. 8. Regnum Veg. 120:1-693.
Imai. S. 1932: Studies on the Hypocreaceae of Japan I. Podostroma. - Trans. Sapporo Nat. Hist. Soc. 12: 114-118.

Karsten, P. 1892: Fragmenta mycologica. 38. - Hedwigia 31: 294-326.

Kornerup, A. \& Wanscher, J.H. 1978: Methuen Handbook of Colour. Ed. 3. - Eyre Methuen Ltd., London.

Lindau, G. 1897: Hypocreales. - In: Engler, H.A. \& Prantl, K.A.E, (eds.), Die natürlichen Pflanzenfamilien 1 (1): 343-372. Verlag W. Engelman, Leipzig.

Liu, P.-G. \& Doi Y. 1995: The Hypocreaceae of China 1. Hypocrea pezizoides with pale green conidia from southern Yunnan, China. - Bull. Nat. Sci. Mus. Tokyo, ser. B, 21: 179-188.

Penzig, O. \& Saccardo, P.A. 1904: Icones Fungorum Javanicorum. - E.J. Brill, Leipzig.

Persoon, C.H. 1800: Observationes Mycologicae. II. Petrum Phillippum Wolf, Leipzig.

Rogerson, C.T. \& Samuels, G.J. 1992: New species of Hypocreales (Fungi, Ascomycetes). - Brittonia 44: 256-263.

Rossman, A.Y. 2000: Towards monophyletic genera in the holomorphic Hypocreales. - Stud. Mycol. 45: 27-34.

Rossman, A.Y., Samuels, G.J., Rogerson, C.T. \& Lowen, R. 1999: Genera of Bionectriaceae, Hypocreaceae and Nectriaceae (Hypocreales, Ascomycetes). - Stud. Mycol. 42: 1-248.

Samuels, G.J. \& Lodge, J. 1996: Three species of Hypocrea with stipitate stromata and Trichoderma anamorphs. - Mycologia 88: 302-315.

Samuels, G.J. \& Rogerson, C.T. 1986 ('May 1984'): New ascomycetes from Amazonas. - Acta Amazonica Supl. 14(1/2): 81-93.

Samuels, G.J. \& Seifert, K.A. 1987: Taxonomic implications of variation among anamorphs. - In: Sugiyama, J. (ed.), Pleomorphic fungi: The diversity and its taxonomic implications: 29-56. Kodansha, Tokyo.

Seaver, F.J. \& Chardón, C.E. 1926: Scientific survey of Porto Rico and the Virgin Islands. 8(1).Botany of Porto Rico and the Virgin Islands. Mycology. - New York Academy of Sciences, New York.

SPSS 2000: Systat 10. Statistics 1. - SPSS Inc., Chicago. Tubaki, K. 1958: Studies on the Japanese Hyphomycetes. V. Leaf and stem group, with a discussion of the classification of Hyphomycetes and their perfect stages. - J. Hattori Bot. Lab. 20: 142-244. 